\title{
Review Article \\ The THz Spectrum of Density Fluctuations of Water: The Viscoelastic Regime
}

\author{
Alessandro Cunsolo \\ Photon Sciences Directorate-Brookhaven National Laboratory, P.O. Box 5000, Upton, NY 11973, USA \\ Correspondence should be addressed to Alessandro Cunsolo; acunsolo@bnl.gov
}

Received 28 November 2014; Accepted 13 January 2015

Academic Editor: Jörg Fink

Copyright ( 2015 Alessandro Cunsolo. This is an open access article distributed under the Creative Commons Attribution License, which permits unrestricted use, distribution, and reproduction in any medium, provided the original work is properly cited.

\begin{abstract}
Relevant advances in the knowledge of the water dynamics at mesoscopic scales are reviewed, while mainly focusing on the contribution provided by high resolution inelastic X-ray scattering (IXS). In particular it is discussed how the use of IXS has improved our understanding of viscoelastic properties of water at $\mathrm{THz}$ frequencies. This specifically involves some solid-like features such as the onset of shear wave propagation, a sound velocity surprisingly similar to the one of ice, and an anomalously low sound absorption coefficient. All these properties can be explained by assuming the coupling of $\mathrm{THz}$ density fluctuations with a structural relaxation process connected to the breaking and forming of hydrogen bonds (HBs). This review also includes more recent IXS results demonstrating that, upon approaching supercritical conditions, relaxation phenomena in water gradually lose their structural character becoming essentially collisional in character. Furthermore, GHz spectroscopy results on supercooled water, suggesting the occurrence of a structural arrest, are discussed. An overview of the new opportunities offered by next generation IXS spectrometers finally concludes this review.
\end{abstract}

\section{Introduction}

Although water covers $71 \%$ of the Earth's surface and is vital for all known forms of life, it remains one of the least understood materials. Liquid water's unique properties and chameleonic nature seem to fit ideally into the requirements for life as can no other molecule. The small size of its molecule belies the complexity of its structural and dynamic behavior both at macroscopic and shorter distances. One of the main peculiarities of water is the highly directional hydrogen bond (HB) interaction between its molecules. These form a network with an intrinsically "open" (high free volume) tetrahedral symmetry, as opposed to the essentially "close packed" interactions in noble gases. Such an intrinsically open arrangement allows for important structure modifications even with moderate density variations and is believed to be the main reason for many well-known thermodynamic and transport anomalies. This makes, in principle, water more prone to structural polymorphic changes at moderate pressures, which are well known to occur in ice [1]. The coexistence of two polyamorphic phases of water characterized by distinct density and local structure is the core assumption behind the hypothesized presence of a second critical point in the supercooled phase of water [2]. Due to the complexity of molecular interaction and structure, it may not be surprising that the dynamics response of water is, again, anomalous. For instance, the pressure dependences of the viscosity [3] and diffusion coefficient are characterized, respectively, by a minimum and a maximum at about $200 \mathrm{MPa}$; in particular the former was observed both at macroscopic [4] and mesoscopic [5] scales. A phenomenological explanation of this anomalous behavior is usually given assuming two competing effects of hydrostatic pressure on the dynamics of water. In one sense, it causes a weakening of the rigid tetrahedral arrangement of the hydrogen network, which, due to the overlap of electron orbitals, is brought about by the increased repulsion between non-hydrogen-bonded neighbors at close contact. This effect contrasts the reduction of the free volume at high pressure, which instead increases the resistance to diffusive flow. At pressure higher that the minimum position, viscosity increases upon increasing density, which is the usual behavior of normal fluids.

No less anomalous is the temperature dependence of viscosity, characterized by a divergence in the supercooled 
phase [6], which corresponds to a similar behavior of the structural relaxation times $[7,8]$, as discussed in Section 7.3 in further detail.

Aside of the unique macroscopic transport properties, also the mesoscopic collective dynamics of water molecules have been the focus of controversial interpretations, which attempted to explain the intriguing similarities between liquid and solid phase responses. The anomalous character of such similarities has been later questioned by several works [9-14], which interpreted them as a manifestation of viscoelasticity, a property that water shares with many other liquids and in particular with glass-forming materials.

This paper will provide a general overview of the current knowledge of the collective dynamics of water at mesoscopic scales, emphasizing the rich phenomenology of high frequency acoustic propagation and its coupling with a structural relaxation process.

\section{The Mysterious Case of "Fast Sound" in Water}

In the mid-seventies the debate around the $\mathrm{THz}$ dynamics of water received substantial motivation from a molecular dynamics (MD) work by Rahman and Stillinger [15]. This work provided an explicit computation of the correlation functions of both longitudinal and transverse components of centers of mass velocities of water's molecules, customarily referred to as longitudinal and transverse currents, respectively.

Compared with previous computer simulations in monatomic fluids, the case of water presented the challenge of a more complex molecular interaction. To approximate it Rahman and Stillinger used a model consisting in the socalled symmetric tetrad of point charges [16]. This is composed of the sum of a Lennard-Jones potential accounting for the centers of mass interactions of neighboring molecules plus a Coulomb interaction term between each couple of four point charges placed in the vertexes of a perfect tetrahedron. Given the complexity of such an interaction, it may be not surprising that the spectrum of velocity-velocity correlation function calculated by Rahman and Stillinger presented some unexpected features with respect to what previously found in Lennard-Jones [17-19] or hard sphere [20] systems, namely,

(1) a double peak structure of the inelastic wings of the density fluctuations spectrum;

(2) a high frequency mode propagating with velocity roughly comparable with the speed of phonons in ice;

(3) a propagating mode dominating the transverse currents, which demonstrated the ability of water to support a shear wave propagation, a finding unprecedented for a simple liquid.

A typical spectrum simulated in [15] is reported in Figure 1, which clearly show the double-peaked structure of the inelastic part of the spectrum.

Another interesting result of [15] concerns the mean square force, whose value was found nearly two orders of magnitude higher than what typically computed for

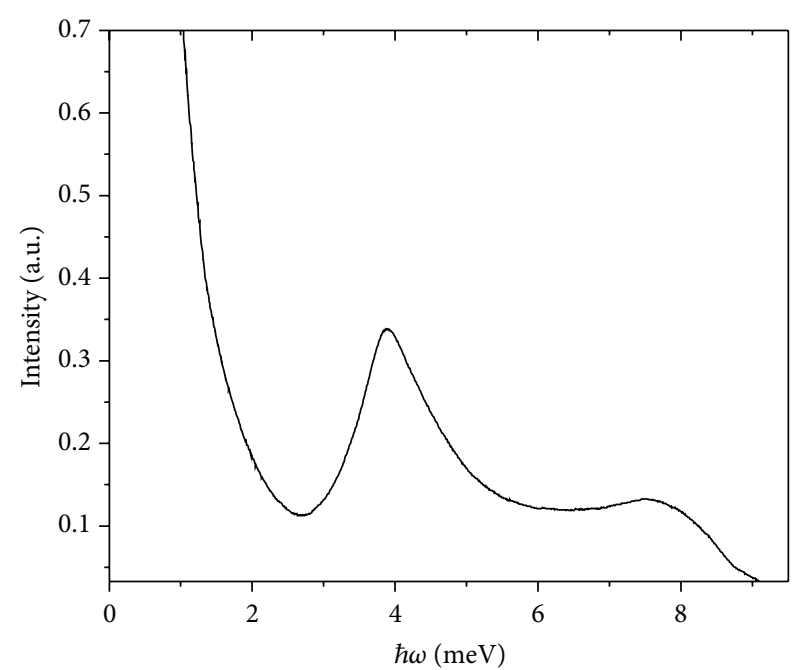

FIGURE 1: The double-peak structure of the spectrum of density fluctuations of water as derived in the computational work of Rahman and Stillinger [15] and at $Q=3.34 \mathrm{~nm}^{-1}$.

monatomic fluids. This anomalously high value was ascribed to the stiffness of the $\mathrm{HB}$ interaction, which caused large fluctuations of the mean force even with small local rearrangements of the molecules. However, the main drawback of the Rahman and Stillinger work was the limited computational power available back at those times, which permitted following only a small number of molecular trajectories (216). This ultimately resulted in a limited access to low exchanged momentum $(Q)$ values. In fact, to preserve the density $\rho \approx 1 \mathrm{~g} / \mathrm{cm}^{3}$, as appropriate for water at nearly ambient conditions, a cubic box of small size $L$ had to be considered, which restricted the probed $Q$ range to $Q \geq$ $Q_{\text {MIN }}=2 \pi / L=3.3 \mathrm{~nm}^{-1}$.

In [15] the sound velocity could be evaluated only at the lowest probed wavevector $\left(Q_{\mathrm{MIN}}\right)$, where the low frequency mode in the spectrum of density fluctuations appeared as a well-resolved peak. Its value derived from the mode shift $\left(\omega_{s}(Q)\right)$ as $c_{s}=\omega_{s}\left(Q_{\text {MIN }}\right) / Q_{\text {MIN }}$ was found to be $\approx 1800 \mathrm{~m} / \mathrm{s}$, that is, only slightly higher than the sound velocity of liquid water at $T=283 \mathrm{~K}$. This suggests interpreting such a low frequency mode as the finite $Q$ extension of the normal hydrodynamic sound mode.

It is worth noticing that the access to lower $Q$ values would have been of crucial importance for the simulation work. It would have clarified, for instance, down to what $Q$ value the double peaked structure of water spectrum persists and how it gradually evolves to the hydrodynamic Brillouin triplet.

The low and high frequency peaks in the water spectra computed by Rahman and Stillinger were thereafter referred to as the "normal sound" and the "fast sound" modes, respectively. As discussed below, successive experimental and computational findings proved the misleading character of this nomenclature. However, it will be used in the remainder of this paper for mere "historical" reasons, until experiments proving its inadequacy $[9,12-14]$ will be explicitly discussed. 
The results obtained by Rahman and Stillinger stimulated new computational and experimental efforts aiming at unraveling the origin of such a peculiar dynamic response. This is, for example, the case of a successive simulation work performed by Impey et al. [21], which mimicked interactions between water molecules with the so-called Matsuoka, Clementi, and Yoshmine (MCY) potential [22]. This model potential essentially includes all terms accounting for atomatom interactions complemented with the electrostatic forces resulting from placing an electron charge, $e$, at the oxygen position and a charge $-2 e$ at a suitable distance along the main symmetry axis of the molecule. The simulation covered a large $T$ range $(242 \mathrm{~K} \leq T \leq 360 \mathrm{~K})$, but only a limited number of molecules could be considered (125). The study aimed at a complete characterization of both orientational and translational motions of water molecules and ultimately derived the values of dynamic parameters relevant to interpret NMR, Raman, incoherent neutron scattering, and dielectric relaxation results. Based on the analysis of molecular centers of mass translations, the authors confirmed the presence of two main peaks at nearly $7.4 \mathrm{meV}$ and $22 \mathrm{meV}$, as previously observed by both Raman and infrared spectroscopy [23]. They assigned these features to center of mass molecular vibrations, respectively, perpendicular and parallel to the molecular plane. Interestingly, the two peaks could be associated to the low and high frequency modes in the $S(Q, \omega)$ simulated by Rahman and Stillinger.

From the experimental side, two inelastic neutron scattering (INS) measurements on liquid $\mathrm{D}_{2} \mathrm{O}$ were performed in 1978 and 1985. The first [24] was executed using a triple axis spectrometer at the low flux source of the $1 \mathrm{MW}$ Casaccia reactor near Rome, Italy. The second [25] was instead performed with the three-axis spectrometer IN8 at the Institut Laue-Langevin in Grenoble, France. One of the main motivations for using triple axis spectroscopy was the inconsistency of previous time of flight (ToF) measurements of the water spectrum reported in the literature [26]. These mutual discrepancies were particularly evident at low frequencies, probably as a consequence of the interpolation procedure used to derive the constant $Q S(Q, \omega)$ from $T o F$ spectra.

These and similar INS experiments used heavy water as a sample, since the highly incoherent INS cross-section of hydrogen would have prevented to measure, from the scattering of $\mathrm{a}_{2} \mathrm{O}$ sample, the coherent part of spectrum. This is ultimately the variable of interest here, since it provides insight into the collective dynamics of the sample.

The experiment of Bosi et al. spanned a limited $Q$ range $\left(Q \geq 5 \mathrm{~nm}^{-1}\right)$ and was performed with a $36.4 \mathrm{meV}$ incident energy, a value ideally suited to resolve the low frequency mode in the spectrum, yet unfit to cover the "fast sound" mode as well, owing to kinematic constraints of neutron spectroscopy (see, e.g., [27, pages 63-101]). This limitation is clearly apparent in Figure 2, where the dispersion of the "fast sound mode" is compared with the parabolic boundaries of the dynamic ranges accessible by Bosi's and Teixeira's measurements.

As compared with the experiment of Bosi et al., the one of Teixeira et al. was performed at a substantially higher incident

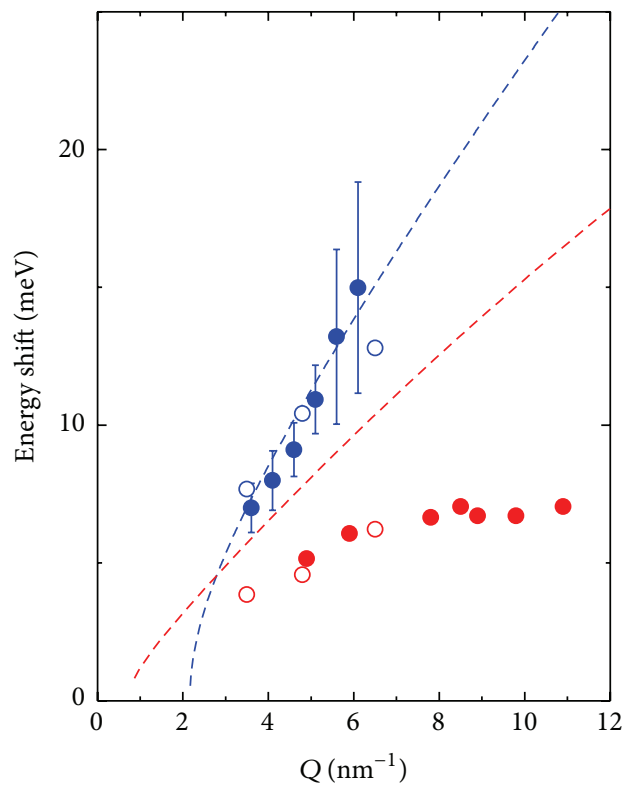

FIgure 2: The dispersion curves of the low frequency (red dots) and high frequency (blue dots) modes in the spectrum of $\mathrm{D}_{2} \mathrm{O}$ are reported as measured by INS in [24] and [25], respectively. The boundaries of the $(Q, E)$ range accessible by the two INS measurements are represented by the dashed parabolas of corresponding colors. Open red and blue dots represent, respectively, the dispersion of the low and the high frequency modes, as determined by the MD computation of Rahman and Stillinger [15].

energy $(80 \mathrm{meV})$, which extended the spanned energy range up to barely include the "fast sound" peak position (see Figure 2). However, this extension could only be achieved to the detriment of the resolution bandwidth, which became too large ( $4.75 \mathrm{meV}$ full width at half maximum, FWHM) to properly resolve the low frequency mode.

Overall, the dispersion curves in Figure 2 confirm the double mode structure of water spectrum found in the MD work by Rahman and Stillinger. The results of the latter simulation are also reported in the plot for comparison. Computational and experimental results are in good mutual agreement and both demonstrate that the two dominant modes exhibit a substantially different dispersive behavior. In particular, the $Q$-dependence of the low frequency mode is extremely weak at high/intermediate Qs, while it becomes sharper at the lower Qs. The sound velocity derived from the low $Q$ slope of the dispersion curve measured by Bosi et al. was $1350 \mathrm{~m} / \mathrm{s}$, which is very close to the known value of the adiabatic sound speed of $\mathrm{D}_{2} \mathrm{O}$. This further supported the interpretation of the "normal sound" mode as the finite$Q$ extension of the ordinary, or hydrodynamic, sound mode; however, it seemed at odds with previous INS measurements on monatomic system such as, for example, neon [28] rubidium [29] and lead [30], which instead suggested a large high $Q$-increase of the sound velocity.

Conversely, the Q-dependence of the "fast sound" frequency measured by Teixeira et al. followed a very sharp straight line, with a slope corresponding to a sound velocity of 
nearly $3300 \mathrm{~m} / \mathrm{s}$, a value fully consistent with the "fast sound" propagation speed reported by Rahman and Stillinger.

Teixeira et al. interpreted the "fast sound" mode as a solid-like excitation selectively propagating over strongly hydrogen-bonded local structures of water. This interpretation relied upon three main arguments: (1) the wavelength associated with the "fast sound" mode was found comparable with the typical size of the patches of strongly hydrogen bonded molecules of water; (2) the measured damping of the sound mode was much smaller than that of the ordinary sound mode extrapolated to the same $Q$ range. Such a low absorption coefficient seemed more appropriate for a solidlike rather than liquid propagation medium; (3) the velocity of the sound mode was found comparable with the one of ice; therefore, the sound wave was likely to propagate through solid-like regions existing in water.

A successive INS experiment performed by Bermejo et al. on $\mathrm{D}_{2} \mathrm{O}$ both above and below the melting [31] proposed a slightly different interpretation scheme. In this case the authors used the ToF chopper spectrometer MARI at the Rutherford Appleton Laboratory in Didcot, UK. This instrument was operated at the same incident energy as in Teixeira's experiment, yet with a narrower energy resolution ( $2 \mathrm{meV}$ FWHM). In agreement with the result of Bosi et al., the presence of a "normal sound" mode in the spectrum was suggested, while in contrast with Teixeira's result, no evidence was found for the "fast sound" mode. The latter finding may be related to the high $Q$ values probed by the experiment $\left(\geq 5.5 \mathrm{~nm}^{-1}\right)$, which moved the "fast sound" position to frequencies approaching or even exceeding the boundaries of the accessible dynamic domain. This, however, cannot explain the discrepancy with the result of Teixeira's measurement, which was also hampered by the same problem (see Figure 2); the inconsistency is probably connected to the different spectroscopic techniques employed in the two experiments, that is, triple axis and ToF spectrometry, respectively, for [25] and [31]. In particular, the interpolation procedure needed to determine constant $Q$ spectra from ToF intensity may have yielded questionable results near the boundaries of the accessible $(Q, \omega)$-plane, where indeed the "fast sound" mode located.

From the analysis of their ToF measurement, Bermejo et al. concluded that no high $Q$ increase was detectable in the $\mathrm{THz}$ sound velocity of water. Furthermore, based on the results obtained on ice, the authors questioned the very acoustic character of the "fast sound" mode, for which they rather hypothesized an optical origin.

\section{The "Fast Sound" \\ Phenomenon as a Possible Manifestation of a Viscoelastic Behavior}

As discussed in the previous sections, until the mid-nineties the onset of the "fast sound" mode in the spectrum of density fluctuations has been the focus of controversial interpretations. A popular one ascribed this "fast sound" mode to high frequency density waves selectively propagating over strongly $\mathrm{HB}$ connected regions of water.
As an alternative explanation, the "fast sound" phenomenon in water was interpreted as the signature of a positive sound dispersion (PSD) revealing the onset of a $\mathrm{THz}$ viscoelastic response. From a physical point of view, the PSD mainly consists in a gradual increase of the measured sound velocity upon increasing the exchanged momentum, $Q$, or the frequency, $\omega$, as a result of the coupling with an internal relaxation process. It is important to notice that the experimental validation of this hypothesis would have required the access to $Q$ s lower and/or Ts higher than those covered by the INS experiments of Bosi and Teixeira.

To gain a basic understanding of the physics behind the onset of a PSD, it is important to recognize that acoustic waves, either spontaneous or generated by a scattering event, induce a time-dependent perturbation of the local equilibrium of the sample. As a response, decay channels are activated to redistribute the energy from the acoustic perturbation toward some internal degree of freedom and these energy rearrangements drive the sample to relax to a new local equilibrium. The time needed to restore the equilibrium, that is, the relaxation time, $\tau$, can be identified with the characteristic timescale of the degree of freedom involved in the energy rearrangement. The mere existence of such a "privileged" timescale logically splits the dynamics of the system into two opposite regimes, depending on how the frequency of the acoustic wave, $\omega_{s}$, compares with $1 / \tau$. Specifically,

(i) in the so-called viscous regime, characterized by $\omega_{s} \ll$ $1 / \tau$, the target sample relaxes instantaneously, thus causing the acoustic propagation to take place over successive equilibrium states;

(ii) in the opposite, elastic regime where $\omega_{s} \ll 1 / \tau$, acoustic oscillations are so rapid that all the system's degrees of freedom become too slow to efficiently dissipate their energy. Therefore, the sound wave propagates elastically, that is, virtually with no energy losses.

Owing to the progressive decrease of acoustic dissipation, the energy of the sound wave increases systematically across the viscous-to-elastic (viscoelastic) transition and so does its propagation speed. In a scattering experiment, this is revealed by the gradual $Q$-increase of the measured slope of the sound dispersion (sound velocity), that is, the mentioned PSD effect. One readily recognizes that the coupling of the sound wave with a specific degree of freedom of the system introduces not only a characteristic timescale, but also a typical size. In other words, when a relaxation is active, the measurement of the sound velocity depends on the probed distance or, equivalently, on the probed $Q$ value. An example of the sharp frequency dependence of sound velocity induced by an active relaxation is reported in Figure 3, which refers to the case of the glass former glycerol [32].

The plot compares independent results obtained with techniques covering disparate frequency windows such as ultrasound (US) absorption [43], spanning the $10-150 \mathrm{MHz}$ range, Brillouin (visible) light scattering (BLS) and Brillouin ultraviolet scattering (BUVS) [32], spanning the $1.5-20 \mathrm{GHz}$ 


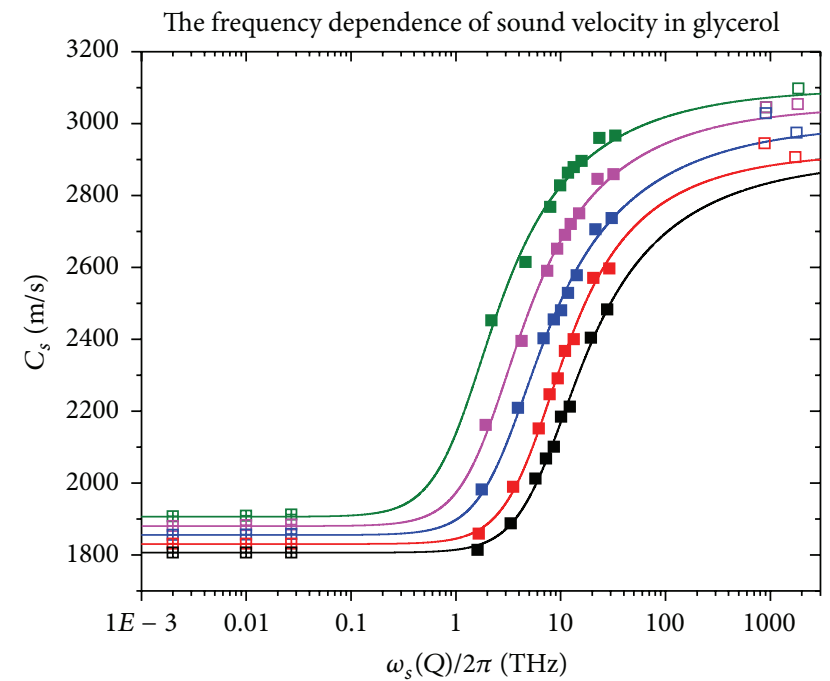

$$
\begin{aligned}
& \text { US BLS IXS US BLS IXS } \\
& \begin{array}{c}
\text { data data data } \\
\boxplus \quad \square T=297 \mathrm{~K} \quad \text { data data data } \\
\square \quad \square \quad T=328 \mathrm{~K}
\end{array} \\
& \text { 田 } \square T=308 \mathrm{~K} \quad \text { 田 } \square \quad \square=338 \mathrm{~K} \\
& \text { 田 } \square T=218 \mathrm{~K}
\end{aligned}
$$

FIGURE 3: The sound velocity of glycerol measured at different frequencies by the spectroscopic techniques and at the sample temperatures indicated in the legend (from [32]).

range, and, finally, inelastic X-ray scattering (IXS) accessing the $\mathrm{THz}$ region. One readily notices that the value of sound velocity dramatically increases upon increasing the frequency or, equivalently, the exchanged momentum.

The inflection point of the dispersion curve, that is, the center of the viscoelastic crossover, locates around a $Q^{*}$ value, at which $c_{s}\left(Q^{*}\right) \approx 1 /\left(\tau Q^{*}\right)$. There, the wave-vector of the scattering-generated acoustic wave $Q^{*}$ becomes comparable with $2 \pi / d$, where $d$ is the size of the degree of freedom involved in the relaxation mechanism. Another remarkable feature emerging from Figure 3 is that upon increasing the temperature, owing to the decrease of $\tau$ the inflection point clearly moves towards higher frequencies (higher Qs). This trend is paradigmatic of the broad class of relaxation processes customarily referred to as structural relaxations, which are characterized by strongly temperature-dependent timescales. Indeed the effects of structural relaxations on the sound propagation can be alternatively characterized by changing the temperature while keeping constant $Q$. In this case the changed variable rather than being a parameter of the probe $(Q)$ is a physical property of the sample, that is, the relaxation time.

Finally and perhaps more importantly, when mesoscopic scales are considered, all transport parameters of the sample need to be generalized as appropriate $Q$-dependent variables. This Q-dependence reflects the local character of mesoscopic transport processes, that is, their sensitivity to the details of the spatial (structural) arrangement of molecules. The complex interplay between dynamical and structural effects has a nontrivial influence on the dispersion curve. In fact, the bending upwards of $\omega_{s}(Q)$ due to viscoelastic effects (positive sound dispersion) overlaps, at intermediate Qs, with the competing bending downwards (negative sound dispersion) caused by the destructive interference of the acoustic wave with the pseudoperiodicity of the structure.

To properly disentangle these dynamic and structural effects, it is often useful to determine the $T$-dependence of transport parameters. In fact changes of temperature, owing to their influence on viscosity, have a stronger impact on transport properties than on the local structure of molecules.

Until the first high resolution IXS spectrometer was first ready for operation on 1995, no experiment could prove the occurrence of a viscoelastic behavior of water over mesoscopic scales. However, some suggestion could be found in the MD simulations and Brillouin light scattering (BLS) measurements to be discussed in the next two sections.

\section{Some Computational Evidence on the Presence of a Positive Sound Dispersion (PSD) in Liquid Water}

The MD simulation by Wojcik and Clementi [44] clearly pointed to a close link between the "fast sound" phenomenon and a high frequency viscoelastic behavior of water. In this work, intermolecular interactions in water were approximated by a MCY potential accounting for the "two-body" term either with or without the addition of a "three-body" one, whose main parameters were determined by $a b$ initio methods. This computation extended the $Q$ range previously investigated up to fully cover the $2.5 \mathrm{~nm}^{-1} \leq Q \leq 49.6 \mathrm{~nm}^{-1}$ range, definitely better suited to observe the possible onset of viscoelastic effects. As a result, the presence of a large PSD was confirmed; furthermore, its amplitude was observed to enhance upon including the three-body term to the modeling of molecular interactions. However, no evidence was reported for the low energy peak either computed or measured in previous literature works.

The enhancement effect that the three-body potential has on the PSD is particularly meaningful since this kind of interaction broadens the spatial range of attractive intermolecular forces. Its observation suggests that the amplitude of PSD intimately depends on the strength of long range attractive forces, as those induced by the HB network.

The authors attempted a description of density fluctuations in the framework of the generalized hydrodynamics theory, based on the idea that the "fast sound" represents the elastic limit of the ordinary hydrodynamic acoustic mode.

Further simulation works seemed to confirm this interpretation, although they sometime explored slightly different approaches to study and interpret the phenomenon. In particular, the work of Ricci et al. [45] was inspired by the circumstance that both a "fast sound" mode and a "double peaked" structure were previously found in the MD simulated spectrum of $\mathrm{Li}-\mathrm{Pb}$ mixture [46]. These were theoretically interpreted $[47,48]$ as a general dynamic feature of binary mixtures having disparate mass' components.

Although water is obviously different from a mixture, the disparate masses of its constituent atoms may make it a suitable candidate for the observation of a mode splitting phenomenon such as the one predicted for binary systems. 
Consistently with the mixture-like scenario, the authors proposed a "two-variable" description of the water spectrum which allowed them to explicitly compute partial density fluctuations of water selectively involving either the hydrogen or the oxygen atomic species.

The MD simulation was carried out by approximating the intermolecular interactions of water with a transferable interaction potential with four points (TIP4P) [49]. This model mimics the oxygen interactions by Lennard-Jones potentials complemented by Coulomb interactions between point charges located at the protons and oxygen sites including a dummy charge $e$ placed along the bisector of the $\mathrm{H}-$ $\mathrm{O}-\mathrm{H}$ angle. The $\mathrm{Q}$ dispersion of acoustic modes dominating simulated spectra had the characteristic double branched structure. The $Q$-dependence of the high frequency mode was found to be roughly consistent with the one previously observed for the "fast sound" mode. The presence of an additional low frequency mode weakly dispersing at high Qs seemed instead to be consistent with the low frequency mode reported by Bosi et al., whose $Q$ dispersion is illustrated in Figure 2.

The analysis of the partial scattering contributions led the authors to conclude that the high frequency mode appears in both (hydrogen and oxygen) partial velocity correlation spectra, revealing its connection to centers of mass molecular movements. Most importantly, it was observed that, at low Qs, this mode transforms into the ordinary sound mode. A further mode selectively involving hydrogen atoms was also observed to appear in the spectrum of density fluctuations and ascribed to librational motions. However, its connection with the "fast sound" mode was excluded, due to both its high frequency $\left(\omega>50 \mathrm{ps}^{-1}\right)$, largely exceeding the one of "fast sound," and the form of its $Q$ dispersion, which suggested an optical rather than acoustic origin.

In a successive theoretical and computational work [50], Ricci et al. proposed a description of density fluctuations in water in terms of the first-order memory function theory. As a result, the presence of two collective excitations could be observed at wavelengths comparable with intermolecular distances.

However, only the high frequency mode was observed to survive in the hydrodynamic regime, where it propagates with a speed comparable to the adiabatic sound velocity of water. The "fast sound" phenomenon was thus connected to a strong PSD leading to the gradual transition between the hydrodynamic and the high frequency regimes. Furthermore, it was demonstrated that the same low frequency mode, previously observed by MD computations and INS experiments, appears in the spectrum beyond some Q-threshold. This mode was associated with molecular centers of mass translations, yet not directly connected with the ordinary adiabatic sound mode, at odds with what hypothesized in $[15,24,31]$.

When accounting for the origin of the large PSD observed in the high frequency mode, the authors invoked two alternative hypotheses.

(1) The first assumed a merely dynamic origin of the PSD, which would represent a manifestation of viscoelasticity, as extensively discussed above. However, this interpretation was clearly in conflict with previous low frequency ultrasound (US) absorption [33], acoustic levitation [51], and Brillouin light scattering (BLS) $[52,53]$ results (to be discussed below) which seemed to confute the presence of a PSD. A possible reconciliation with these results was suggested by previous depolarized light scattering (DLS) measurements [35], showing that relaxations in water are active in ps window, well below the sensitivity of both BLS and US.

(2) Alternatively, the authors considered the possibility that the observed PSD could have a static, rather than dynamic, origin. According to this hypothesis, the PSD could have been induced by the negative low $Q$ slope of the static structure factor, as evidenced by both previous [54] and successive [55-57] small angle scattering measurements. In fact, within the reasonable assumption that, at finite Qs, the sound velocity is proportional to $Q / S(Q)^{1 / 2}$; a negative slope of $S(Q)$ thus translates into a more-than-linear increase of the sound dispersion. Although this argument may provide a plausible explanation of the observed PSD, it does not account for the circumstance that the observed $Q$ increase of $S(Q)$ is too weak to substantially contribute to the large PSD observed in water.

The connection of the "fast sound" mode with center of mass translations is one of the most important outcomes of the MD work of [50]. This conclusion was further developed in a successive theoretical and computational work by Balucani et al. [58]. In this work the dynamic behavior of water was compared with that of a monatomic system, that is, cesium near the melting. As opposite to other monatomic systems such as liquid Ar, liquid Cs is characterized by relatively soft interatomic interactions. Most importantly, both in water and in liquid Cs, the specific heats ratio $\gamma$ is close to 1 , which implies a negligible coupling of the sound dispersion with thermal diffusion (see (7) in Section 7.2).

In [58] it was found that PSD effects are much larger in water than in cesium and this was attributed by authors to the harsher intermolecular repulsions of water. In fact, a larger PSD is generally reported for materials with harder interparticle repulsions, such as water and argon, rather than for those having a soft interatomic potential, such as liquid metals [27].

The key parameter identified by the authors as a reliable measure of the amplitude of PSD is the squared Einstein frequency $\Omega_{0}$, related to the second space derivative of the potential and providing a rough measure of the cage oscillation frequency of a liquid [27]. In particular, it was found that $\Omega_{0}$ is nearly 8 times larger in water than in cesium.

The work of Balucani et al. enabled the clear observation of a single mode dominating the longitudinal current spectrum; however, its frequency was found to be sensibly higher at high Qs than reported by previous literature measurements and calculations (see Figure 4). Furthermore, no evidence was found for the "normal sound" peak reported 


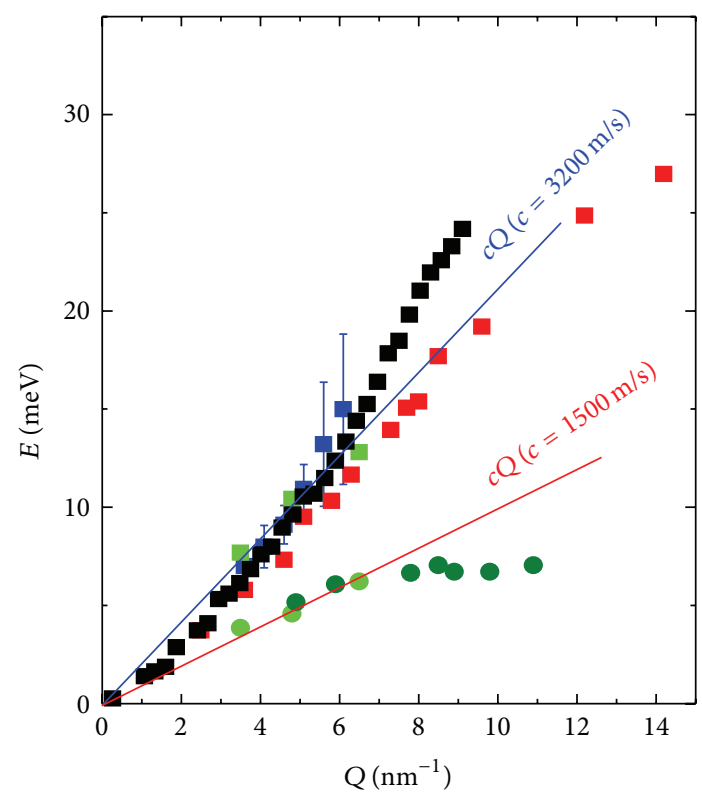

- MD (Rahman and Stillinger, 1974)

- MD (Rahman and Stillinger, 1974)

- INS (Bosi et al., 1978)

- INS (Teixeira et al., 1985)

- MD (Balucani et al., 1993)

- MD (Sciortino and Sastri, 1994)

FIGURE 4: The sound dispersion curves of water as determined before the advent of IXS by the experimental and computational methods indicated in the legend. The two linear laws corresponding to the hydrodynamic and "fast sound" dispersions are reported for reference as red and blue straight lines, respectively.

by previous works, while the presence of a single peak was confirmed dominating the transverse current spectra and having frequencies similar to those indicated in literature investigations.

The work of Balucani et al. confirmed that the longitudinal sound mode exhibits a clear PSD when Q moves from the hydrodynamic to the "fast sound" regime and that this crossover can be consistently predicted by the generalized hydrodynamic theory.

A successive joint MD and normal mode analysis [59] extended the exchanged momentum probed by previous MD simulations on water down to $Q=0.26 \mathrm{~nm}^{-1}$, while using a TIP4P potential to model intermolecular interactions. In this work the unambiguous observation of viscoelastic effects on the dispersion curve of water was made possible by the access to substantially lower Qs opened up by improved computational capabilities. The $T$-dependence of the PSD was found to be consistent with the hypothesis of a structural relaxation process. The normal mode analysis evidenced instead that the $\mathrm{O}-\mathrm{O}-\mathrm{O}$ intermolecular bending mode had a sizable influence on the longitudinal sound dispersion. The latter was interpreted as a general behavior that water likely shares with other associated liquids.

In Figure 4 a comparison is proposed between some of the computations and experimental results discussed above.
All data are in a fairly good mutual agreement, although the values computed by Sciortino and Sastry [59] clearly exceed the "fast sound" frequency reported by other works. This likely owes to the large amplitude of the PSD at the low temperature considered in such a simulation (270 K).

\section{Evidence of a PSD in Supercooled Water Gained by Lower Frequency Techniques}

As discussed in previous sections, toward the mid-nineties of the past century, the experimental knowledge of the $\mathrm{THz}$ dynamics of water was at an impasse. In fact, any further advance would have required the development of new generation inelastic spectrometers with less stringent kinematic limitations and accessing to lower $Q$ values.

However, even before such spectrometers were finally available, the presence of a large PSD in water was experimentally detected by several spectroscopic methods probing $(Q, \omega)$ much lower than those typical of the viscoelastic crossover $(Q \approx 2 \pi / \lambda, \omega \approx 1 / \tau)$, namely, (i) ultrasonic (US) absorption, covering the $10 \mathrm{KHz}-100 \mathrm{MHz}$ frequency window and wave-vector roughly ranging in the $10^{-8}-10^{-4} \mathrm{~nm}^{-1}$ interval [37, 39, 51]; (ii) Brillouin light scattering (BLS), covering the $1-10 \mathrm{GHz}$ frequency window with exchanged $Q \mathrm{~s}$ spanning the $10^{-2} \mathrm{~nm}^{-1}$ range $[7,52,53,60,61]$.

These measurements needed to cope with major technical challenges, since deep supercooling conditions had to be reached to move $\tau$ closer to their window of sensitivity; however even the lowest $T$ practically accessible $(\approx 247 \mathrm{~K}), \tau$ can at best attain $\mathrm{a} \approx 10$ ps value, still too small for a proper detection by BLS and even less by US.

As a matter of fact, in the various low $Q, \omega$ measurements on water reported in the literature, the quantitative determination of $\tau$ often required audacious $T$ - or $\omega$-extrapolations and/or the mixing with a more viscous solvent. The latter strategy was followed by an US work [37], in which the relaxation time of pure water was determined by extrapolating US results for increasingly diluted aqueous solutions of glycerol. However, the literature reports also an US work on pure water [33] indicating the presence of "anomalous" dispersive effects in the sound absorption.

Conversely and somehow surprisingly, the results of some early BLS works on water questioned the mere presence of a PSD. This owed, once again, to the scarce visibility of the sought-for effect when probed by $\mathrm{GHz}$ spectroscopy, unless a deep supercooling of the sample was achieved.

Specifically, no evidence of a viscoelastic behavior was reported in the BLS measurements of Rouch et al. [52, 53], nor in a successive work [62] comparing BLS results and previous acoustic levitation ones [51]. Conversely, the BLS work of Conde et al. [62] suggested the occurrence of a slight negative sound dispersion since the hypersonic sound velocity measured by BLS was found to be slightly lower than the one previously measured by US. Such an apparent frequency decrease of sound velocity raised some interest; however, as later shown $[63,64]$, it had no correlation with the dispersive behavior of water. It rather followed from the circumstance that the sound velocity measured by BLS 


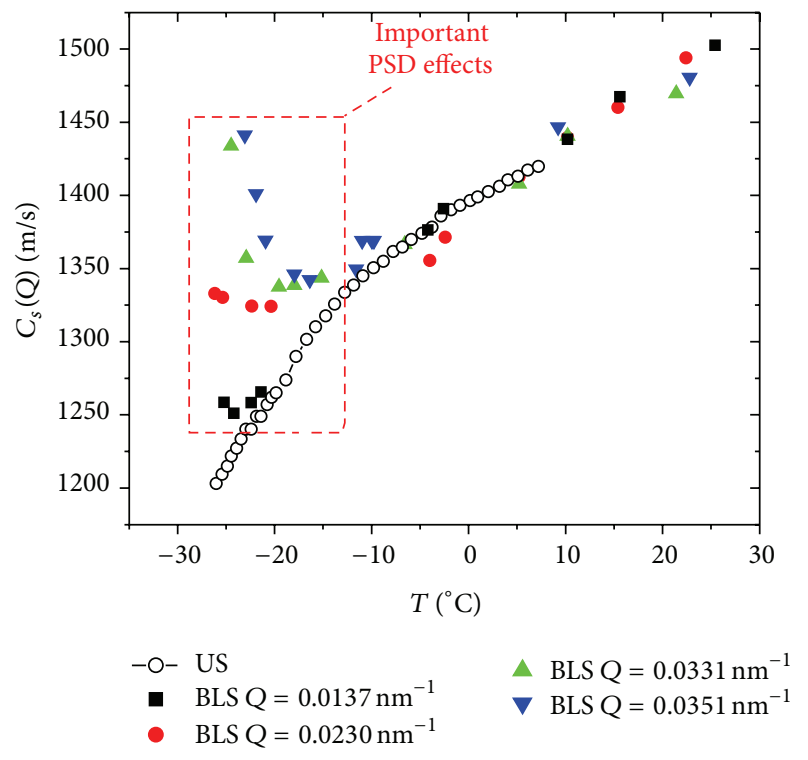

FIgure 5: The $T$-dependence of the sound velocity is reported as measured by US ([33]) and by BLS ([7]) at the indicated $Q$ values in both normal liquid and supercooled water.

(hypersonic velocity) is expectedly lower by few percent than the counterpart measured by US (ultrasonic velocity), unless competing viscoelastic effects are relevant. The latter is the case of deeply supercooled water (see, e.g., Figure 5) for which the PSD induced by the viscoelasticity largely outweighs such an "apparent" negative dispersion.

The search for a PSD and related relaxation phenomena in water was also challenged by previous dielectric relaxation measurements $[65,66]$, demonstrating the existence in water at ambient condition of a single dielectric relaxation time spanning the $n s$ window, which matches the domain covered by BLS.

Scientists in the field were baffled by the circumstance that such a dielectric relaxation did not correspond to a "mechanical" or structural relaxation observable in the BLS spectrum.

However, this apparent "inconsistency" between dielectric and spectroscopic results was easily explicable. In fact, the presence of a dielectric relaxation does not imply the one of a structural relaxation in the same time window. In general dielectric and mechanical (structural) relaxations are linked to distinct rearrangement mechanisms and are characterized by disparate timescales [67]. Specifically, molecular electrical dipoles are allowed to rearrange their directions only after the relaxation of the local HB structure is fully accomplished. This implies that the timescale of structural relaxation, $\tau$, is, in general, shorter than that of dielectric relaxation. Therefore, the presence of a dielectric relaxation right in the ns domain probed by BLS suggested that the structural relaxation was likely active only at shorter timescales.

In order to move relaxation processes to a more accessible time window, successive BLS experiments mostly aimed at probing deep supercooling conditions.

In particular, Magazú et al. [60] successfully used BLS to detect a clear PSD in supercooled water and ascribed it to the same structural relaxation previously observed by US techniques [37]. A viscoelastic modeling of the frequency dependence of sound velocity enabled the authors to derive a $\tau$ value in the ps window. The essentially Arrhenius behavior [68] of $\tau$ corresponded to an activation energy of $18 \mathrm{kcal} / \mathrm{mole}$, a value substantially larger than both the HB energy [23] and the activation energy derived from viscosity $[39,69]$ and Raman scattering $[23,35]$ measurements.

Further physical insight into the structural relaxation of water was gained in a successive BLS work [7], in which the signatures of such a phenomenon were sought for by modeling the shape of Brillouin spectra based upon the hypothesis of a single relaxation timescale. As a result, the presence in water of a relaxation with a merely structural character was confirmed. Its Arrhenius behavior corresponded to a $5.2 \mathrm{kcal} / \mathrm{mole}$ activation energy, a value consistent with that reported in previous literature results. Figure 5 illustrates PSD effects on the sound velocity of supercooled water as measured by BLS in [7] as a function of T. The US measurements [33] of the corresponding quantity are also included for reference.

One clearly notices that the access to deep supercooling conditions is associated with the onset of a substantial Qincrease of sound velocity (i.e., a PSD), as expected when a relaxation phenomenon becomes active.

\section{First IXS Studies on Liquid Water and Ice}

The inherent limitations which have hampered $\mathrm{THz}$ spectroscopy until the mid-90s could be successfully overcome after the development of the first high resolution IXS spectrometer: ID16 beamline at the European Synchrotron Radiation Facility (ESRF) in Grenoble, France. A detailed description of this spectrometer and a general account of IXS technique go beyond the scope of the present review and can be found, for example, in [70,71].

In the early operation phase of ID16 three consecutive experiments on water were carried out with a gradually improved resolution bandwidth: $5 \mathrm{meV}$ [9], $3.5 \mathrm{meV}$ [11], and $1.4 \mathrm{meV}[12]$.

As compared to the INS measurements performed by Teixeira and Bosi, these first high resolution IXS measurements slightly extended the spanned $Q$ region, from 3.6$6 \mathrm{~nm}^{-1}$ to $2-10 \mathrm{~nm}^{-1}$. More importantly, the virtual lack of kinematic limitations also enabled a substantial extension of the spanned frequency range.

Among various results, these early IXS measurements demonstrated the following.

(i) The "fast sound" frequency measured either in $\mathrm{H}_{2} \mathrm{O}$ (by IXS) or in $\mathrm{D}_{2} \mathrm{O}$ (by INS) is identical to that within the error bars, as clearly appears from Figure 6(a). This finding seems to confute the hypothesis that the "fast sound" mode selectively propagates through the hydrogen atoms, rather suggesting that the whole water molecule is involved in the motion. In fact, in the latter case, the discrepancy in sound velocity caused by the mass difference between $\mathrm{H}_{2} \mathrm{O}$ and $\mathrm{D}_{2} \mathrm{O}$ molecules is expected to be smaller than the actual 


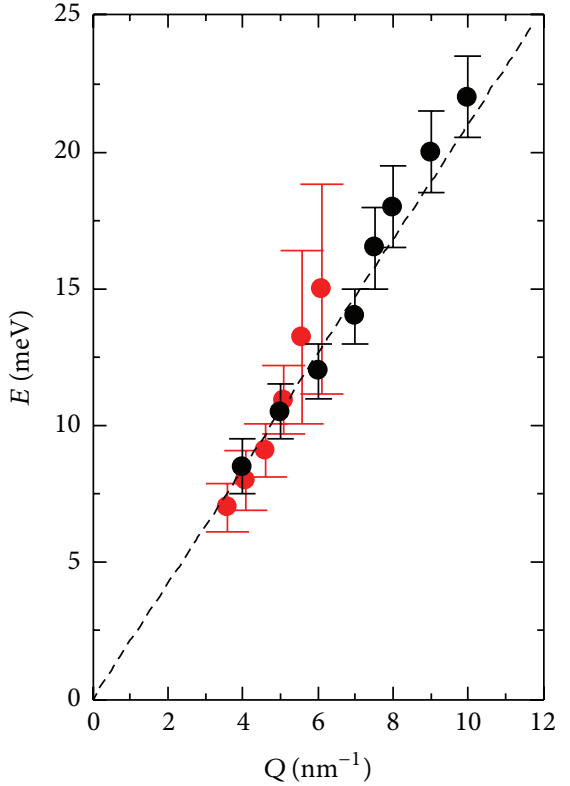

(a)

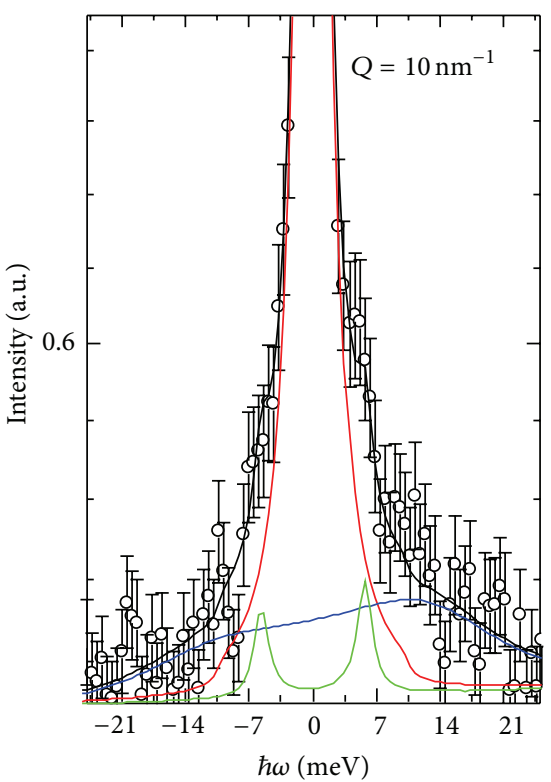

(b)

FIGURE 6: (a) Comparison between the dispersion curves of the "fast sound" mode as determined by INS [27] (red dots) and IXS [9] (black dots). The linear best fit of IXS data is also reported for reference as a dashed straight line. (b) A typical raw spectrum (open circles) measured by IXS [12] at $Q=10 \mathrm{~nm}^{-1}$ (open circles) along with its best fitting of (1) the total line-shape (black line), (2) the quasielastic contribution (red line), and (3) the low (green line) and (4) the high (blue line) frequency inelastic modes.

error bars of INS and IXS experiments, as suggested by experimental results. Conversely, if the mode had selectively involved individual hydrogen (for IXS) or deuterium (for INS) atoms, the relative mass difference would have caused a visible discrepancy in the sound velocities measured either in the $\mathrm{D}_{2} \mathrm{O}$ or in the $\mathrm{H}_{2} \mathrm{O}$ samples.

(ii) A clear PSD can be observed in water at mesoscopic scales, in agreement with some previous MD results as, for example, $[44,45,59]$. Most importantly, the new IXS measurements indicated that the zero and infinite frequency sound velocities derived from the dispersion curve are comparable with those of liquid water and ice, respectively. This further substantiated the hypothesized link between the "anomalous" sound dispersion and the onset of a viscoelastic behavior.

(iii) The presence of an additional low frequency peak in the water spectrum was confirmed for $Q$ larger than $4 \mathrm{~nm}^{-1}$. Such a peak can be clearly recognized by looking at the spectrum in Figure 6(b) (green line). The broad shoulder (blue line) dominating the high frequency spectral tails can be instead identified with "fast sound" mode.

(iv) Further insight into the $\mathrm{THz}$ dynamics of water was gained with the IXS work of [11], based on a comparative measurement of the water spectrum above and below the melting. To check for possible anisotropies of sound propagation in ice, the authors considered different hexagonal ice samples, both in poly- and single-crystal forms, and compared the sound speeds measured along different crystallographic directions. The absence of any significant anisotropy was then ascribed to the almost perfect tetrahedral coordination of ice molecules. It was observed that a single sharply dispersive longitudinal acoustic branch dominated the IXS spectra of ice in the first Brillouin zone, while in the second an additional low frequency and weakly dispersive mode showed up. Based upon previous lattice vibrations calculations and INS measurements [72], this further spectral feature was assigned to a transverse optical (TO) mode. Consistently with previous INS $[24,31]$ and successive IXS [73-76] measurements, a similar low frequency peak was found also in liquid phase and, in analogy to the case of ice, it was ascribed to the coupling of the spectrum with shear waves. The soundness of this assignment was confirmed few years later by both MD computations [77] and IXS measurements [73]; this point will be discussed in some detail in a forthcoming publication. One of the advantages in performing IXS measurement on a solid sample was, of course, the presence of much better resolved inelastic peaks in the spectrum. A demonstration of that is provided by Figure 7, which illustrates the $Q$ evolution of the IXS spectra of ice measured in [11]. The circumstance that the TO mode can only be observed in the IXS spectrum of ice for $Q$ values beyond the boundary of first Brillouin zone $\left(\approx 7.5 \mathrm{~nm}^{-1}\right)$ follows from selection rules valid for both X-ray and neutron scattering. However, for 


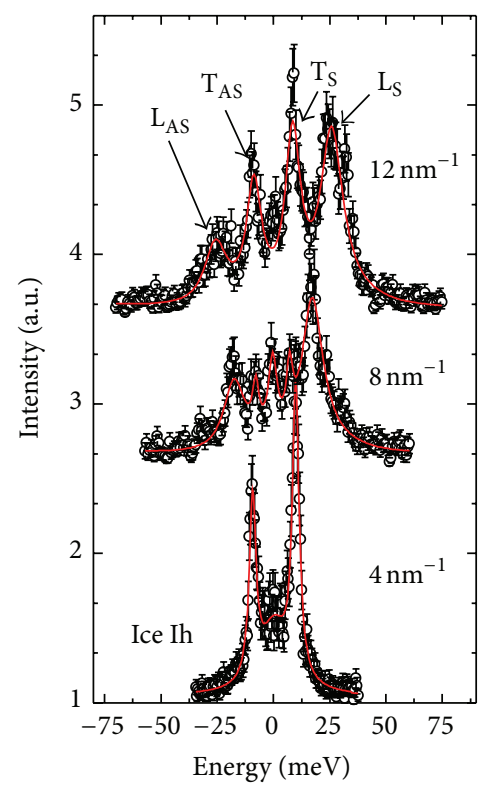

FIGURE 7: The spectrum of polycrystalline ice Ih measured in [11] at some selected Qs (open circles) is compared with corresponding best fit model line-shapes. The letters "T" and "L" label, respectively, the transverse and longitudinal peaks, while the suffixes "S" and "AS" indicate, respectively, the "Stokes" and "anti-Stokes" components.

an amorphous system as liquid water, the absence of a large-scale symmetry makes in principle a transverse, or shear, mode observable at lower $Q$ values.

(v) Another important finding of first IXS measurements is the anomalously narrow width of the fast sound mode. This confirms previous INS [25] results and indicates an abrupt decrease of sound absorption upon approaching the elastic limit.

Resuming, although viscoelasticity is an almost ubiquitous property of fluids, early IXS measurements on water demonstrated that few unique features were associated with the viscoelastic behavior of this system, such as the hypothesized coupling of density fluctuation with shear waves, the large amplitude and frequency window of the PSD, the low value of sound absorption, and the similarity of the high frequency sound speed in liquid and solid phases.

This similarity is clearly represented by Figure 8 which compares several sound dispersion measurements performed in both liquid and solid phases of water until 1996. It can be readily noticed that, at high Qs, the dispersion curves in liquid and solid phases are nearly indistinguishable, at least to within the relatively large error bars.

On a general ground, it can be concluded that in both phases density fluctuations propagate through the rigid network structure formed by HBs. When probed over timescales much shorter than the HB lifetime the local structure of water molecules appears essentially frozen and somehow similar to the one of ice. If, conversely, much longer timescales are probed, many $\mathrm{HB}$ rearrangements of the HBs occur, which makes allowance for the liquid-like diffusive flow take place.

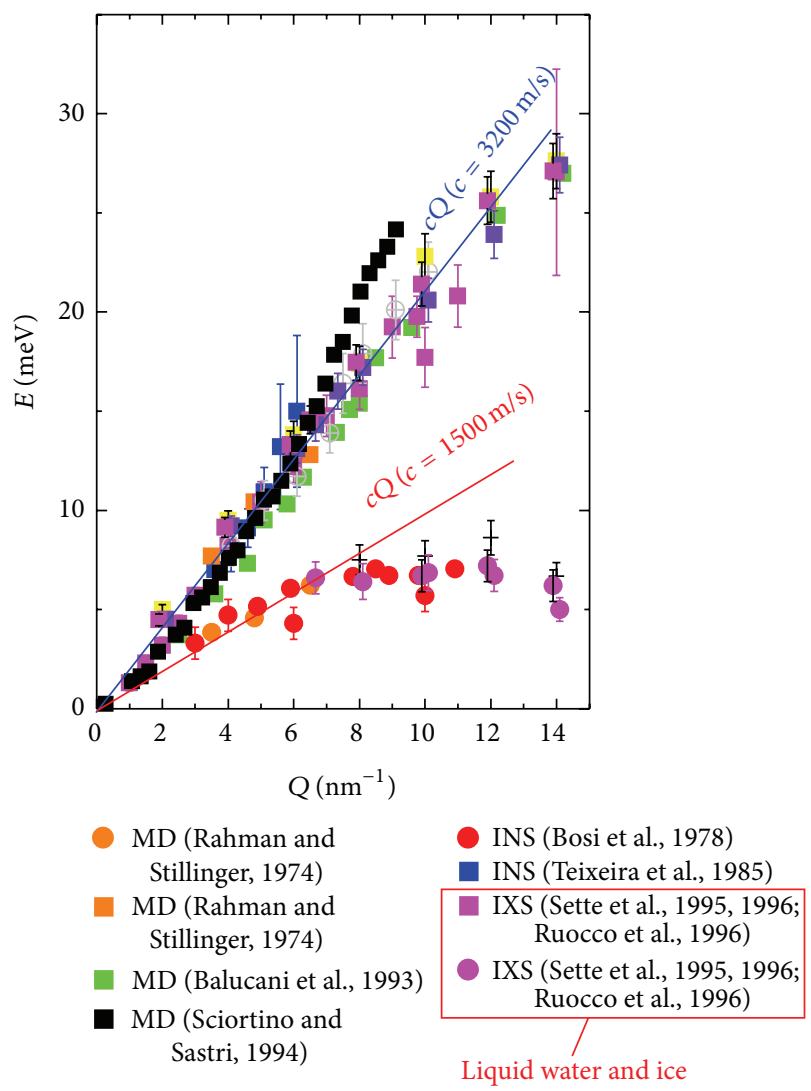

FIGURE 8: Sound dispersion curves of the high frequency (squares) and low frequency (circles) modes in the spectrum of water and ice as determined in the various experiments and simulations indicated in the legend.

\section{Evidence of a Structural Relaxation in the IXS Spectrum of Water: Some More Quantitative Insight}

As extensively discussed in Section 3, the solid-like response of water dynamics at $\mathrm{THz}$ frequencies can be explained assuming a viscoelastic behavior induced by the presence of a relaxation process.

Two successive IXS works performed in 1999 demonstrated the soundness of this hypothesis also shedding a deeper insight into the underlying relaxation phenomenology. Specifically, the first work [14] proposed an analysis of both $T$ and $Q$ dependencies of the longitudinal moduli measured by IXS demonstrating the presence of a structural relaxation with a single, or at least a dominant, timescale. The second work [13] addressed a more detailed and informative modeling of the spectral shape by using a model derived within the memory function formalism and shedding direct insight on the $T$ and $Q$ evolution of relevant relaxation parameters.

The two works covered a rather extended $T$ interval ( $273 \mathrm{~K} \leq T \leq 473 \mathrm{~K}$ ), with the pressure being adjusted to keep the density as close as possible to $1 \mathrm{~g} / \mathrm{cm}^{3}$, as prescribed by the equation of state (EoS) [38]. The choice of moving 

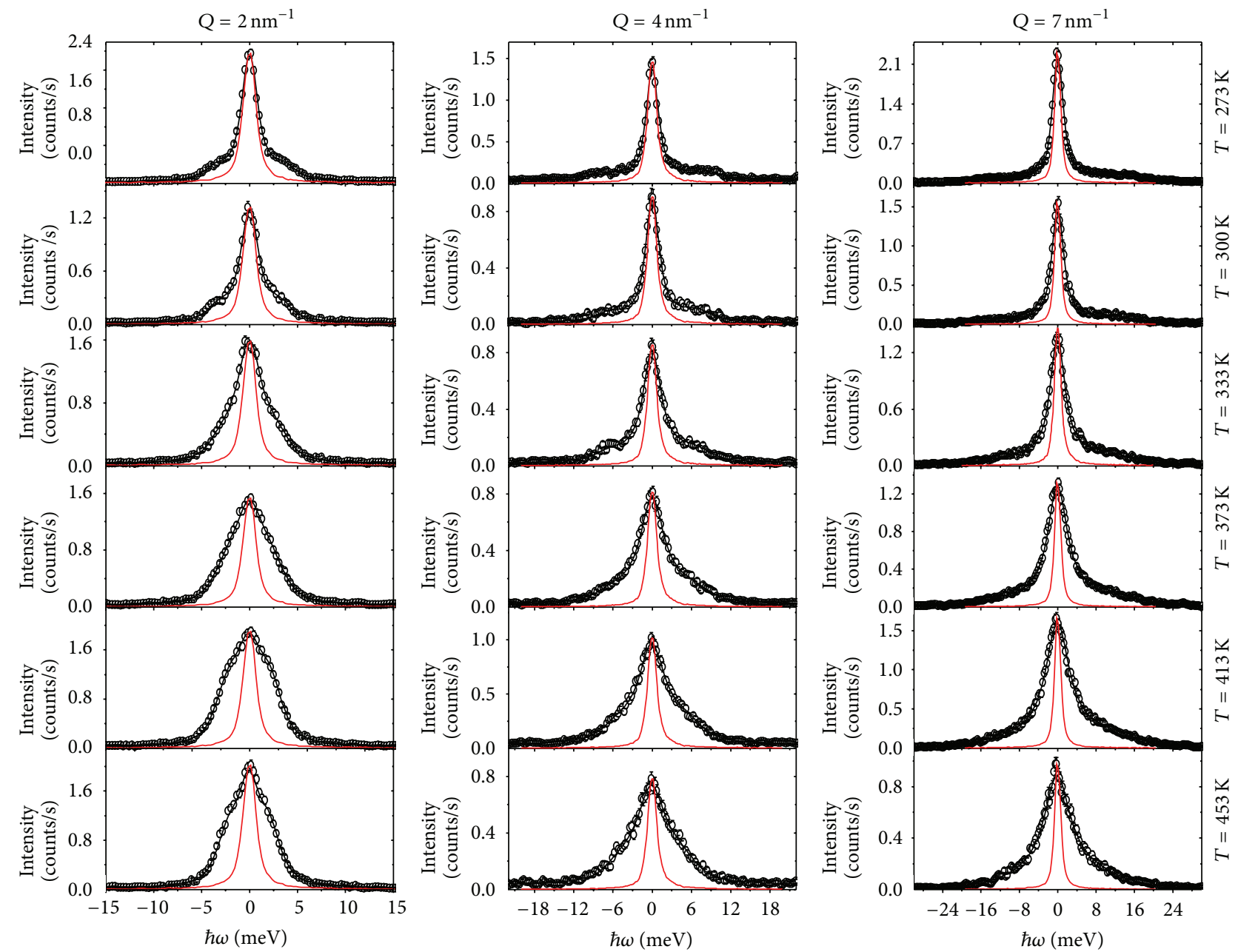

FIGURE 9: Typical IXS spectra measured in liquid water at the indicated temperatures and exchanged momenta (open circles). The measurements are compared with the fits performed with the experimental resolution function (red line), normalized to the maximum intensity of the IXS shapes.

the sample along a nearly constant density path was adopted to minimize the influence of free volume variations on the relaxation process. The relatively low $Q$ values probed by the experiment $\left(2 \div 7 \mathrm{~nm}^{-1}\right)$ made the authors safely discard the spectral contribution from the additional low frequency inelastic mode, previously associated with the coupling of the spectrum with shear waves. In fact, this mode was found to have a nonnegligible spectral contribution mostly at Qs and Ts higher than those typically probed in [13].

Figure 9 displays a selection of IXS spectra of water measured in $[13,14]$.

It clearly appears that the inelastic shoulders become more and more pronounced as the temperature is increased, which mostly reflects the corresponding decrease of the quasielastic relaxational contribution, often referred to as the "Mountain peak" [78]. In fact, being relaxations essentially frozen at low $T \mathrm{~s}$, their spectral contribution concentrates at $\omega \approx 0$; conversely at high $T$ s they become extremely rapid and their very broad spectral distribution gradually merges into the spectral background.
7.1. Relaxation Phenomena Studied through IXS Longitudinal Moduli. When probing the viscoelasticity of a material, the longitudinal modulus $M=\rho c_{s}^{2}$ is often a useful variable to deal with.

Within the so-called Debye approximation, its time dependence-or, equivalently, the one of viscosity-takes the form of a simple exponential decay [79] and its Fourier transform $M(\omega)$ thus reads as

$$
M=M_{\infty}-\frac{M_{\infty}-M_{0}}{1+i \omega \tau} .
$$

One readily notices that, upon increasing the frequency, the longitudinal modulus moves from its viscous limiting value, $M(0)=M_{0}=\rho c_{0}^{2}$, to the elastic one, $M(\infty)=M_{\infty}=$ $\rho c_{\infty}^{2}$. As mentioned above, in a spectroscopy experiment such frequency-dependence is usually revealed by a corresponding $Q$-dependence, induced by the $Q$ increase of the sound 


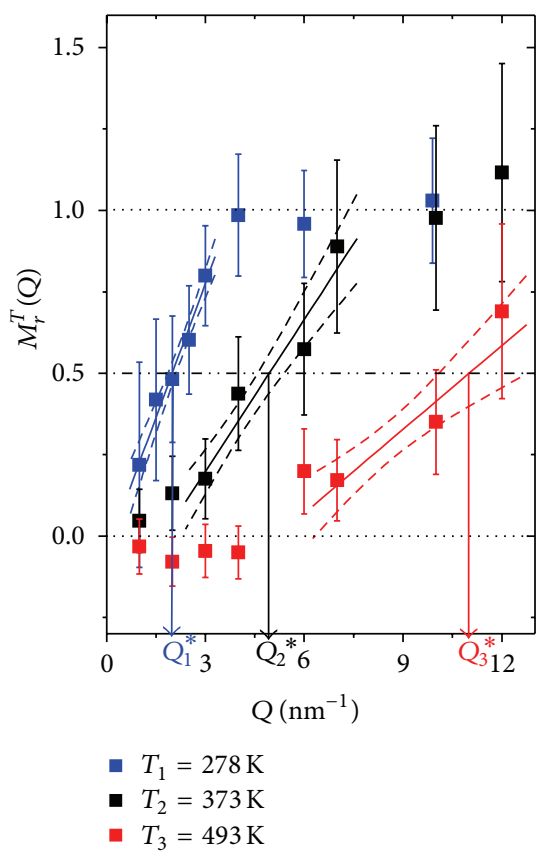

Figure 10: The reduced longitudinal modulus of water measured by IXS [14] as a function of $Q$ and at the $T$ values indicated in the legend. The centers of the viscoelastic crossover $Q^{*}$ are indicated by horizontal arrows. The straight lines used for the interpolation of the viscoelastic crossover positions are reported (solid lines) together with the curve corresponding to $a \pm 1 \sigma$ error (dashed line).

velocity $c_{s}$. Often, when studying relaxation phenomena, it is particularly useful to deal with the dimensionless variable

$$
M_{r}=\frac{M-M_{0}}{M_{\infty}-M_{0}}=\frac{c_{s}^{2}-c_{0}^{2}}{c_{\infty}^{2}-c_{0}^{2}}
$$

customarily referred to as the reduced longitudinal all parameters appearing in (1) and (2) are $Q$ and $T$ dependent variables. What makes $M_{r}(Q)$ a convenient variable to deal with is the fact that it does not depend on density and it simply changes from 0 to 1 as $Q$ moves from the viscous to the elastic regime. Most importantly, within the single timescale approximation, one has $M_{r}\left(Q^{*}\right)=0.5$, where $Q^{*}$ is the center of the relaxation crossover at which $\omega_{s}\left(Q^{*}\right) \approx 1 / \tau\left(Q^{*}\right)$. The viscoelastic transition of the reduced modulus can be observed while keeping $Q$ constant and varying $T$, or vice versa, changing $Q$ at constant $T$. While going from low to high Qs (low to high Ts), the reduced modulus increases from 0 to 1 , that is, from the viscous to the elastic value.

An IXS measurement of the reduced longitudinal modulus of water is discussed in [14] as obtained either at constant $Q$ or at constant $T$; the results of these measurements are reported in Figures 10 and 11, respectively. As clearly shown in Figure 10 (Figure 11), in such a work, the $Q$ (the $T)$ position of the viscoelastic crossover was determined by using $M_{r}^{T}\left(Q^{*}\right)\left(M_{r}^{\mathrm{Q}}\left(T^{*}\right)\right)=0.5$. The corresponding $Q^{*}$ and $T^{*}$ values are indicated by vertical arrows in the figures.

Once the crossover centers $Q^{*}$ and $T^{*}$ were determined by interpolation, the $\tau\left(Q^{*}\right)$ and $\tau\left(T^{*}\right)$ values could then be

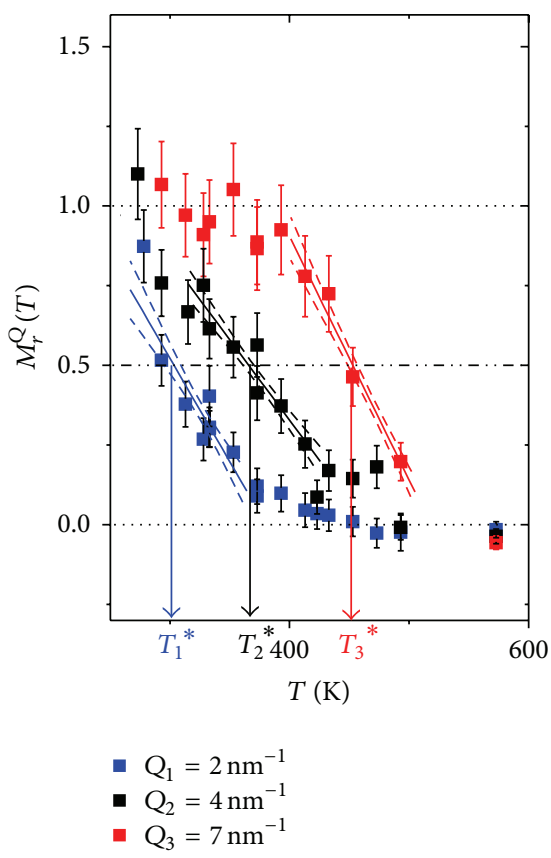

FIGURE 11: The longitudinal reduced modulus measured by IXS [14] as a function of $T$ and at the $Q$ values indicated in the legend. For each curve, the center of the viscoelastic crossover, $T^{*}$, is indicated by a vertical arrow as in Figure 9. The straight lines used for the interpolation of the viscoelastic crossover positions are reported (solid lines) together with the curve corresponding to $a \pm 1 \sigma$ error (dashed line).

extracted from the inelastic shift, $\omega_{s}\left(Q^{*}\right)$ and $\omega_{s}\left(T^{*}\right)$, using the viscoelastic condition $\omega_{s}=1 / \tau$.

It is worth noticing that the actual calculation of the reduced modulus requires in general some additional approximation, since the limiting values of the sound velocity involved in it cannot be easily determined.

Furthermore, at mesoscopic scales transport parameters become local variable; the quantities $c_{0}$ and $c_{\infty}$ in (1) depend on $Q$. In [14] the Q-dependence of $c_{0}$ was assumed to be negligible, while its $T$-dependent value was fixed to the adiabatic sound velocity derived from the EoS of water [38]. Since the $Q$-dependence of $c_{0}(Q)$ is, in first approximation, $\propto 1 / \sqrt{S(Q)}$, neglecting it ultimately amounts to assuming $S(Q)$ nearly flat over the explored $Q$ range. From [54-57] one can estimate the accuracy of this approximation to be better than $\approx 10 \%$ for the measurements discussed in [14]. The determination of $c_{\infty}$ required more drastic assumptions, since its $T$-dependence cannot be derived directly from the EoS. In [14] both the $T$ - and Q-dependencies of $c_{\infty}$ were discarded and their constant value was extracted from the inelastic shift of the IXS spectra measured at $T=278 \mathrm{~K}$, that is, at a temperature sufficiently low to safely assume the elastic regime to be fully reached.

Finally, it should be considered that the value of the apparent sound velocity may generally depend on the model used to approximate the measured spectral shape, especially in the viscoelastic window. However, several attempts made 
in [14] to use models alternative to the DHO did not appreciably change final results.

When comparing the outcome of a $\mathrm{THz}$ relaxation measurement to available lower frequency data, it seems more appropriate to deal with the timescale $\tau_{c}=\left(c_{\infty}^{2} / c_{0}^{2}\right) \tau$. In fact, the determination of $\tau_{c}$ allows the outcome of IXS measurements to be consistent with relaxation measurements probing the hydrodynamic, or continuous, limit. This timescale represents the relaxation time of the compliance $J$, which is linked to the longitudinal modulus through $J=$ $M^{-1}$.

The six values of $\tau_{c}$ derived in [14] as discussed above are reported in Figure 12 and therein compared with the relaxation times extracted from the line-width of the DLS spectrum in $[35,36]$, along with those determined by US absorption [37] and BLS [7]. For recent Brillouin UV scattering (BUVS) measurements [34], although these measurements have been performed along different constant-density paths, those included in Figure 12 refer to the $\rho=1.015 \mathrm{~g} / \mathrm{cm}^{3}$ which is the density value closest to the one probed in [13].

On a rigorous ground, not all spectroscopic techniques considered in Figure 12 measure the same observable. Specifically, the observable probed by DLS is different from the density-density correlation function measured by IXS. However, DLS results are here useful as a reference since it has been shown that in water the DLS spectra mostly couple with the translational dynamics of the molecules through the DID (dipole-induced-dipole) mechanism and that the spectral contribution from rotational dynamics is less than $10 \%$ to the total signal [35]. Figure 12 demonstrates that a good agreement exists between IXS and DLS data.

Conversely, both BLS and US results attain systematically higher values, albeit following a roughly parallel trend. This discrepancy in absolute values can be at least partially explained by recognizing that free volume effects become more relevant in the supercooled phase probed by lower frequency techniques.

The linear Arrhenius plot of $\tau$ suggests that the observed relaxation has an activated character within the probed $T$-window. In [14] the activation energy derived from the Arrhenius trend ( $5.5 \mathrm{kcal} / \mathrm{mole})$ was found to be comparable with the energy of a single $\mathrm{HB}(2.8 \mathrm{kcal} / \mathrm{mole})$ and with the value derived from BLS data in the plot, yet much lower than that reported in $[60,61,63,64]$.

The rough match between the activation energy of $\tau$ and the HB energy one would suggest that the structural relaxation process is somehow connected to the continuous breaking and forming of HBs.

Resuming, the IXS works on water discussed so far clarified that the high frequency sound mode in water is nothing but the elastic limit of a viscoelastic transition. This pointed to the similarity of water with other fluids, since the property of viscoelasticity is commonly believed to be a nearly ubiquitous property of these systems. For this reason, the use of the term "fast sound" was hereafter considered obsolete if not misleading, since it seems to indicate a peculiarity of water rather than a nearly universal feature shared by most liquid systems.

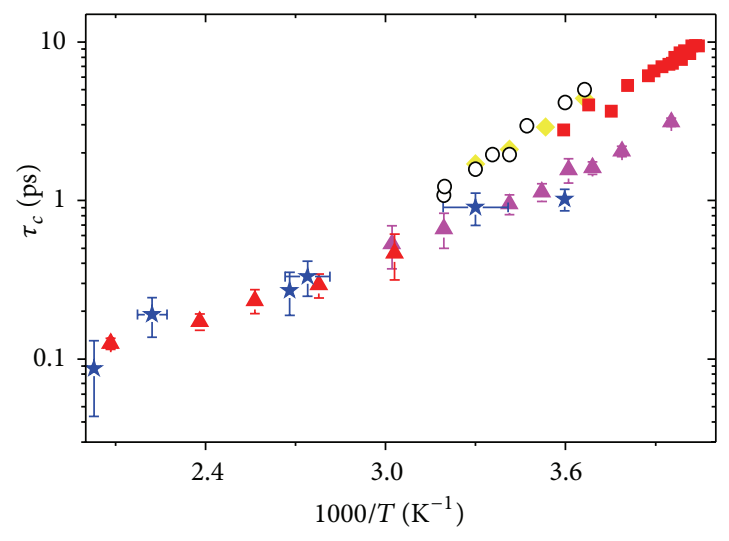

FIGURE 12: Arrhenius plot of the relaxation times of water measured by various spectroscopic techniques: (i) IXS [14] (from the analysis of the reduced longitudinal moduli discussed in the text, blue stars); (ii) BUVS [34] at $\rho=1.015 \mathrm{~g} / \mathrm{cm}^{3}$ (open circles); (iii) DLS from [35] (red triangles) and (iv) [36] (magenta triangles); (iv) BLS [7] (red squares); and (v) US [37] (yellow diamonds).

Perhaps more importantly, these IXS results also stressed the inadequacy of the "normal sound" denomination often used to quote the low frequency peak. In fact this mode, rather than representing the finite $Q$ extension of the ordinary or "normal" sound mode, arises from the coupling of density fluctuations with shear waves, as first suggested by [11] and later confirmed by both MD computations [77] and IXS measurements [73-76].

The IXS spectra measured in the IXS works discussed above were modeled by a simple damped harmonic oscillator (DHO) profile [80] accounting for inelastic excitations, plus a Lorentzian function describing the quasielastic intensity.

It needs to be stressed that the DHO model has some inherent flaws, the main ones being the failure to fulfill the first two sum rules [80] and the limited ability in reproducing the details of the spectral shape in the viscoelastic window.

The main reasons for using a $\mathrm{DHO}+$ Lorentzian profile to model IXS spectra were (i) its inherent simplicity, that is, its ability to provide a straightforward determination of the sound velocity and (ii) the need of keeping consistency with previous INS works, which used a similar model. (iii) Finally, assuming an extremely narrow quasielastic contribution, the used model is analytically isomorph to the hydrodynamic spectrum measured by BLS.

However, owing to the poor ability of this model to approximate the spectrum of liquids in the viscoelastic regime, successive IXS investigations of the structural relaxation of water $[13,57,73-76,81]$ were based on a physically more grounded line-shape description. The relevant formal aspects behind the derivation of such a model are briefly recalled in the next subsection.

7.2. The Viscoelastic Modeling of the Spectrum of Water: Basic Formalism. The variable of interest here is the normalized correlation function of microscopic density fluctuations:

$$
\Phi_{\mathrm{Q}}(t)=\frac{\left\langle\delta \rho_{\mathrm{Q}}(t) \delta \rho_{\mathrm{Q}}^{*}(0)\right\rangle}{\left\langle\left|\delta \rho_{\mathrm{Q}}(0)\right|^{2}\right\rangle},
$$


where $\delta \rho_{Q}(t)$ is the Fourier Q-component of the microscopic density fluctuation $\delta \rho(r, t)$. The dynamic structure factor $S(Q, \omega)$ is defined as

$$
S(Q, \omega)=S(Q) \int_{0}^{\infty} e^{i \omega t} \Phi_{Q}(t) d t
$$

where the static structure factor was introduced:

$$
S(Q)=\left\langle\delta \rho_{\mathrm{Q}}(0) \delta \rho_{\mathrm{Q}}^{*}(0)\right\rangle=\int_{-\infty}^{\infty} S(\mathrm{Q}, \omega) d \omega
$$

which provides direct insight on the static, or structural, properties of the sample. The time dependence of $\Phi_{Q}(t)$ is governed by the well-known Langevin equation [82]:

$$
\frac{\partial^{2} \Phi_{Q}(t)}{\partial^{2} t}+\omega_{0}^{2}(Q) \Phi_{Q}(t)+\int_{0}^{t} m_{Q}\left(t-t^{\prime}\right) \frac{\partial \Phi_{Q}\left(t^{\prime}\right)}{\partial t^{\prime}} d t^{\prime}=0
$$

Here the variables $m_{Q}(t)$ and $\omega_{0}(Q)$ represent, respectively, the memory kernel and the proper frequency of the system. In the frequency domain, the dynamic structure factor can be written as

$$
S(Q, \omega)=\operatorname{Im}\left[\omega^{2}-\omega_{0}^{2}(Q)-i \omega m_{Q}(\omega)\right],
$$

where $\operatorname{Im}[\cdots]$ denotes the imaginary part, while $m_{Q}(\omega)$ is the Fourier transform of the memory function. While modeling the line-shape, it is often convenient to fix the value of $\omega_{0}(Q)$ in (6) and (7) by superimposing to $S(Q, \omega)$ the fulfillment of the first two sum rules, which eventually leads to [83]

$$
\begin{gathered}
M_{0}=S(Q), \\
\omega_{Q}(Q)=\frac{Q v_{T}}{\sqrt{S(Q)}}=\sqrt{\frac{M_{2}}{M_{0}}} .
\end{gathered}
$$

Here $v_{T}=K_{B} T / M$ is the component of the thermal speed along the direction of the exchanged momentum $\vec{Q}$, while $M$ and $K_{B}$ are, respectively, the molecular mass and the Boltzmann constant; finally, $M_{n}=\int_{-\infty}^{\infty} \omega^{n} S(Q, \omega) d \omega$ represents the $n$-order moment of the spectrum.

It can be shown that an expression of $m_{\mathrm{Q}}(t)$ consistent to the hydrodynamic, $Q=0$, limit is given by

$$
m_{\mathrm{Q}}(t)=\omega_{0}^{2}(Q)(\gamma-1) e^{-D_{T} Q^{2} t}+2 \nu_{L} Q^{2} \delta(t),
$$

where $D_{T}$ and $\gamma$ are the thermal diffusivity and the constant pressure-to-constant volume specific heats ratio, respectively, while $\nu_{L}=\left(\eta_{b}+4 / 3 \eta_{s}\right) / \rho$ is the kinematic longitudinal, viscosity with $\rho, \eta_{b}$, and $\eta_{s}$ being the density, the bulk, and the shear viscosity coefficients, respectively. The time-dependent memory function in (9), once Fourier transformed and inserted in (7), allows the line-shape model to reproduce the well-known Brillouin triplet predicted by the hydrodynamic theory.

To derive a suitable expression for the time-dependent memory function beyond the hydrodynamic limit, the second term in (9) must be replaced by a generalized longitudinal kinematic viscosity, $K_{L}(Q, t)$, for which a suitable model needs to be introduced. The simplest ansatz for this function is an exponential decay, customarily referred to as the viscoelastic or Debye model [84]. As opposite to noble gases, in water the viscoelastic model begins to fail when $Q$ approaches the position of first diffraction peak [85], due to the increasing relevance of high $Q$ fast dynamics. A better account of the time decay of $K_{L}(Q, t)$ can be obtained by assuming that it splits into two main contributions.

(i) The first one is related to molecular vibrations in the local environment, which induce a loss of correlation at the very early stage, usually on the $10^{-13}$ s range, and have almost no temperature dependence.

(ii) The second one, instead, corresponds to the structural relaxation routinely observed in glass formers (see, e.g., [86]) and has a strongly $T$-dependent decay.

For an associated fluid as water, the structural term is expected to be largely dominant, at least at the lowest Qs. Furthermore, at ambient temperature or higher, the structural term can provide a contribution to the memory decay which falls right in the region probed by IXS and, in a first approximation, can be modeled by a single exponential decay. Conversely, the fast microscopic decay is often well approximated by a simple Markovian term, that is, a term $\propto \delta(t)$. This option also minimizes the number of free parameters involved in the fitting routine.

Overall, the most common model used in most literature IXS measurements on simple fluids reads as:

$$
\begin{aligned}
& m(Q, t) \\
& =2 \Gamma_{0}(Q) \delta(\tau)+\omega_{0}^{2}(Q)(\gamma(Q)-1) \exp \left(-D_{T}(Q) Q^{2} t\right) \\
& \quad+\Delta^{2}(Q) \exp \left[-\frac{\left(c_{0}^{2}(Q) / c_{\infty}^{2}(Q)\right) t}{\tau_{c}(Q)}\right] .
\end{aligned}
$$

The third term on the right-hand side of (10) is in principle the one describing the phenomenology of a viscoelastic crossover; its amplitude $\Delta^{2}(Q)=c_{\infty}^{2}(Q)-c_{0}^{2}(Q)$ is customarily referred to as the relaxation strength.

In the various viscoelastic modeling of the IXS spectrum of water reported in the literature, several approximations have been adopted, as appropriate to the specific $Q, \omega$ range or thermodynamic conditions considered.

In some cases the thermal contribution in (10) has been either discarded [75], based on the expectation that at high Qs one has $\gamma(Q) \approx 1$ or kept fixed to the value obtained replacing all thermal coefficients with the respective macroscopic (Q-independent) counterparts [13, 74]. This approximation, certainly reasonable at relatively high Ts [87], turns out to be particularly useful since the thermodynamic parameters of water are very well known from the literature. Furthermore, the zero frequency (viscous) limit of sound velocity, can either be assumed as $Q$-independent and coincident to the adiabatic sound velocity $[13,73,74]$ or derived from the first two sum rules of the dynamic structure factor [75] (i.e., through (5)). Ultimately, a model for the classical part of $S(Q, \omega)$ can be readily obtained by inserting (10) into (7). 
All formulas introduced above provide a consistent description of the spectral shape for classical systems only. Quantum effects are handled by imposing to the model the fulfillment of detailed balance, which causes an asymmetry in the spectral shape due to the dependence on $\omega$ of the statistical population of the states of the sample. In various IXS works in the literature this is achieved by multiplying the classical spectral shape by an $\omega$-dependent term:

$$
S_{M}(Q, \omega)=\frac{\hbar \omega}{k_{B} T}[n(\omega)+1] S(Q, \omega),
$$

with $n(\omega)$ being the Bose-Einstein population factor. Finally, a realistic description of the measured spectral shape must include the contribution of the energy resolution function as well as all spurious intensity and background effects. Ultimately, the following model profile is commonly used to fit measured IXS spectral shapes:

$$
I(Q, \omega)=K S_{M}(Q, \omega) \otimes R(\omega)+B .
$$

Here $S_{M}(Q, \omega)$ is the generic model used for the classic part of the dynamic structure factor, while the symbol “ $\otimes$ " represents the convolution operator; finally, $K$ and $B$ are $\omega$-independent terms representing, respectively, an overall scaling factor and a plateau term, including spectral background and electronic noise of the detectors.

A line-shape model similar to the one described above was successfully employed in several IXS experiments on samples as diverse as HB liquids [13, 88], noble gases [89], liquid metals [90], diatomic liquids [91], supercooled molecular systems [92], glasses [93], and even quantum systems [94, 95].

7.3. Relevant Conclusions from the Viscoelastic Analysis of Water Spectra. The ability of the memory function in (7) to reproduce the shape of the IXS spectrum of water is demonstrated in Figure 13. There, the measured spectra are reported against the corresponding best fit line-shapes, the fit residuals being also included in the inset for reference. The logarithmic representation emphasizes the agreement between measured and model line-shapes even in the extreme energy regions reached by the spectral tails.

Such a favorable comparison provides the ground for a reliable discussion of best fit parameters. A thorough analysis of their Q- and T-dependence can be found in [13]; here only few relevant results are summarized.

The Q-dependence of best fit parameters derived from the IXS line-shape can be used to extrapolate their macroscopic $(Q=0)$ limiting values, which was required for a direct comparison with measurements probing the continuous (US) or quasicontinuous (BLS) probes. However, this poses a problem as of the most suitable prediction for these $Q$ dependencies. For instance, the $Q$-dependence of $\tau_{c}(Q)$ in the literature has been approximated by complex models based upon an interpolation between the hydrodynamic and the single particle limits [96]; a simple linear approximation was instead proposed in [13]. Although this assumption has a poor justification considering that the $Q$ derivative of $\tau_{c}(Q)$ must vanish upon approaching the hydrodynamic limit, it becomes accurate enough when small $Q$ values are explored.

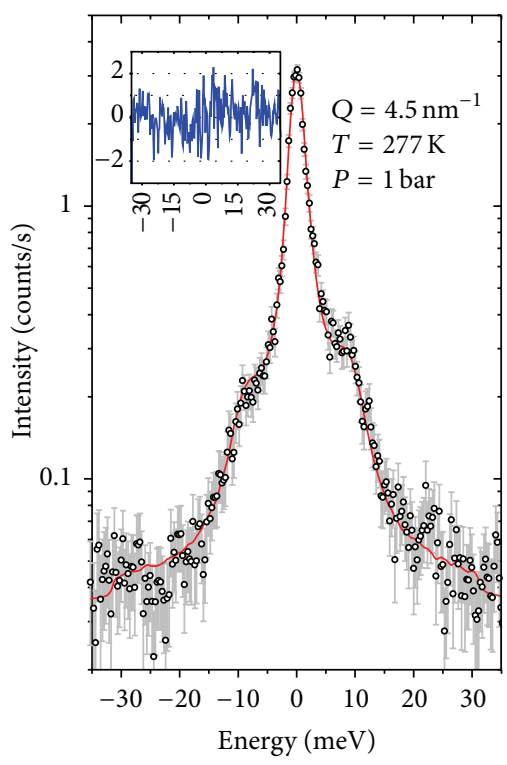

FIGURE 13: A typical IXS spectrum measured in water [13] compared with corresponding best fit line-shape obtained with the memory function in (10) as discussed in the text. The residual of the fit is reported in the inset.

Figure 14 shows the Q-dependent relaxation times of water derived in [13] at selected $T$ values and compares them with corresponding linear best fits. It can be noticed that, at high $T \mathrm{~s}, \tau_{c}(Q)$ decreases substantially, which reflects the corresponding decrease in viscosity, and its Q-dependence becomes correspondingly weaker.

Figure 15 illustrates the temperature dependence of the $Q=0$ extrapolated relaxation times, that is, the $y$-intercept of the best fitting straight lines in Figure 14. For the sake of comparison, the plot also includes the following:

(i) the relaxation times obtained from the analysis of longitudinal moduli discussed in Section 7.1;

(ii) the relaxation times derived from the analysis of DLS spectra [36] corresponding to a density of $\approx 1 \mathrm{~g} / \mathrm{cm}^{3}$;

(iii) the relaxation times derived in another DLS analysis performed at lower Ts [35].

Overall, all data reported in Figure 15 confirm that in the $T$ range between $250 \mathrm{~K}$ and $500 \mathrm{~K}$ the $T$-evolution of the relaxation time is Arrhenius-like.

The activation energy extracted from the Arrhenius trend of IXS data from [13] is $3.8 \mathrm{kcal} / \mathrm{mole}$, in agreement with the activation energy reported in $[23,97,98]$ and with the known value of the HB energy [23].

However, clear discrepancies can be observed between the values derived from the viscoelastic modeling of the lineshape in [13] and those derived from the IXS longitudinal moduli analysis in [14] or from literature DLS data $[35,36]$, both being systematically smaller by about a factor two.

The discrepancy between the viscoelastic modeling of IXS spectrum and DLS results is only partly unexpected, since the 


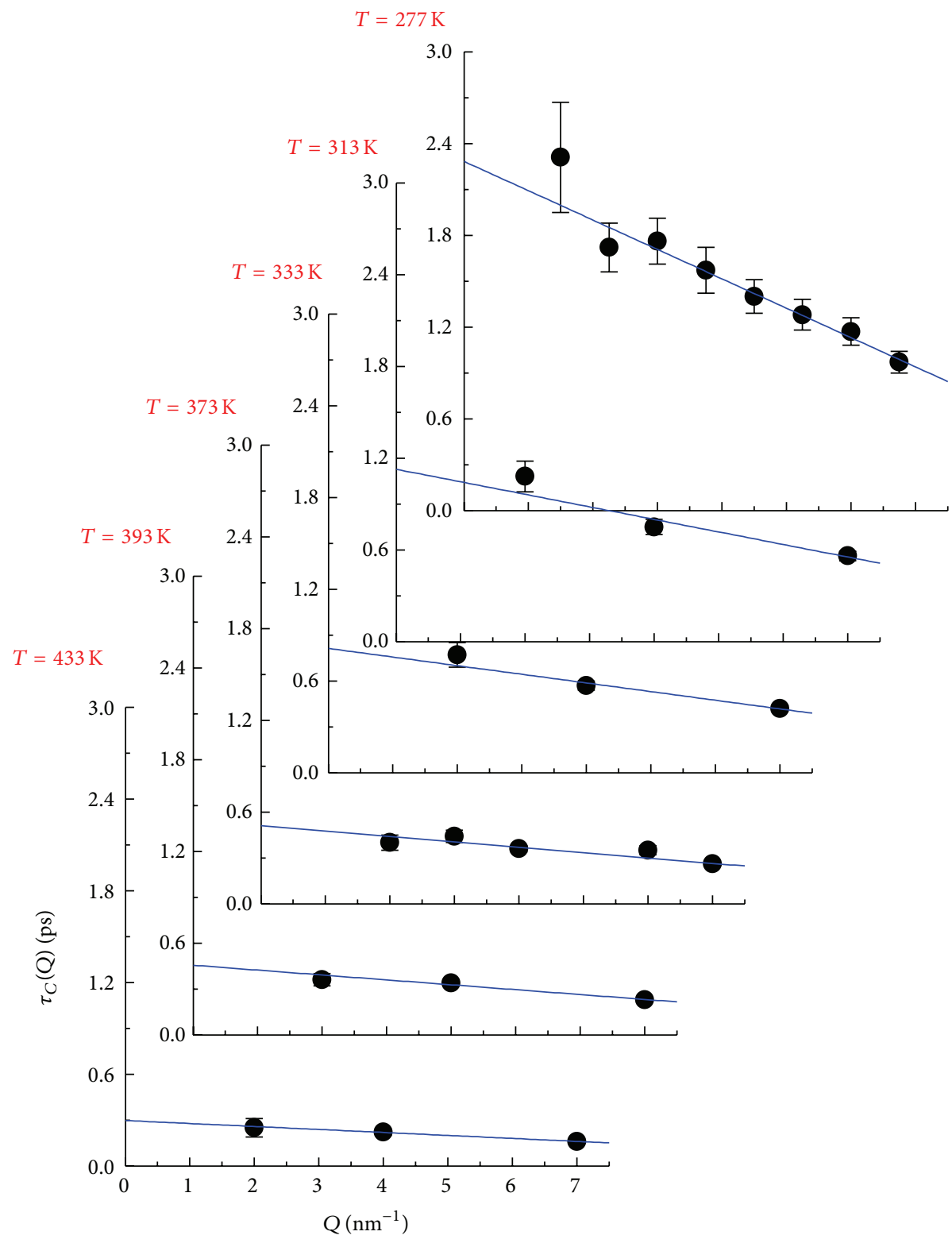

FIGURE 14: The $Q$-dependence of the relaxation timescale $\tau_{c}(Q)$ is reported as derived from [13] at the temperatures indicated in the individual plots. The corresponding linear best fits used to extrapolate the $Q=0$ limits are also reported for reference.

DLS spectrum is essentially the Fourier transform of a fourbody correlation function, which expectedly has a faster time decay than the pair correlation probed by IXS.

The inconsistency with the IXS longitudinal moduli analysis has instead a less obvious interpretation. Some contributing factors can be tentatively identified as follows: (i) the approximation on the zero and infinite frequency sound mode may have failed; (ii) the use of the DHO model may have originated possible overestimates of $\omega_{s}$ or underestimates of $\tau$ (the two options being equivalent since $\omega_{s} \tau=1$ ); or (iii) the single timescale approximation inherent to the longitudinal moduli analysis discussed in Section 7.1 may have caused an underestimate of the relaxation times.

Another transport parameter directly derived from the viscoelastic modeling of IXS spectra is the generalized longitudinal kinematic viscosity, $v_{L}(Q)$, whose $Q$-dependent value can be obtained by imposing a long time $(t \gg \tau)$ consistency between (9) and (10). The macroscopic, $Q=0$, limit of this variable can be then determined as

$$
\begin{aligned}
v_{L} & =\lim _{Q \rightarrow 0} \nu_{L}(Q) \\
& =\lim _{Q \rightarrow 0}\left[\frac{\Gamma_{0}(Q)}{Q^{2}}+\frac{\Delta^{2}(Q)}{\rho}\left(\frac{c_{0}(Q)}{c_{\infty}(Q)}\right)^{2} \tau_{c}(Q)\right] .
\end{aligned}
$$

In [13] some approximations were made on the various $Q$ dependencies of involved parameters while evaluating the limit in (13): (1) as mentioned, $c_{0}$ was approximated by the $Q$-independent value deduced from the EoS; (2) $c_{\infty}(Q)$ was replaced by its $Q$-average, as its best fit values were found to be 


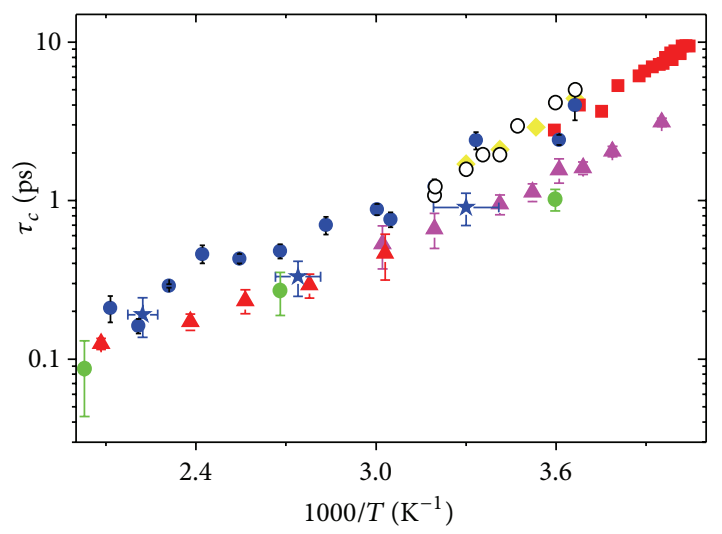

Figure 15: The Arrhenius plot of the relaxation time of water as measured by various techniques. (i) Results from the viscoelastic analysis of the IXS line-shape [13] (blue dots) are compared with the relaxation times reported in Figure 12 with the same symbols.

essentially Q-independent; (3) the first term inside the square brackets in (13) was replaced by a constant since best fit values of $\Gamma_{0}(Q)$ were found to exhibit a $Q^{2}$-growth; and, finally, (4) the $Q$-dependence of $\tau_{c}(Q)$ was approximated by a linear law, as discussed above.

The $T$-dependent values of $v_{L}$ determined in [13] through (13) within the above approximations are reported in Figure 16 in Arrhenius plot. These IXS results combined with previous BLS ones in supercooled phase-also included in the plot-suggest a slight bending upwards of the Arrhenius curve at the lower $T$ s.

Such more-than-linear trend may parallel the VogelTammann-Fulcher (VTF) law [99] characteristic of fragile glass-forming liquids [100], even though in the past a criticallike divergence was proposed instead [101]. On a general ground, a VTF growth can be predicted by assuming that free volume effects influence the viscous flow [102]. Although in [13] these free volume effects were minimized by keeping the density essentially constant, they were certainly more relevant to the BLS measurements on supercooled water reported in Figure 16.

One readily notices that the values of $v_{L}$ determined by [13] follow a temperature evolution essentially parallel to the one of the shear component $v_{s}=\eta_{s} / \rho$, as derived from the EoS [38].

This may suggest that structural relaxations influence shear and bulk viscosities in the same way, which reveals the isotropic character of the underlying relaxation process. This confirms the conclusions of lower frequency US [39] and BLS [7] measurements.

Another important conclusion drawn from the viscoelastic analysis of IXS spectra in [13] concerns the high frequency (elastic) limit of viscosity, $\eta_{\infty}$. Although generally low at moderate $T$ values, the percent variation of $\eta_{\infty}$ was found to increase with $T$, changing from $5 \%$ of the total viscosity at $273 \mathrm{~K}$ up to nearly $40 \%$ at $473 \mathrm{~K}$.

Interestingly, previous BLS results on supercooled water $[7,60]$ and US measurements on water-glycerol mixtures [37] indicated that $\eta_{\infty}$ attains negligibly small values at even lower

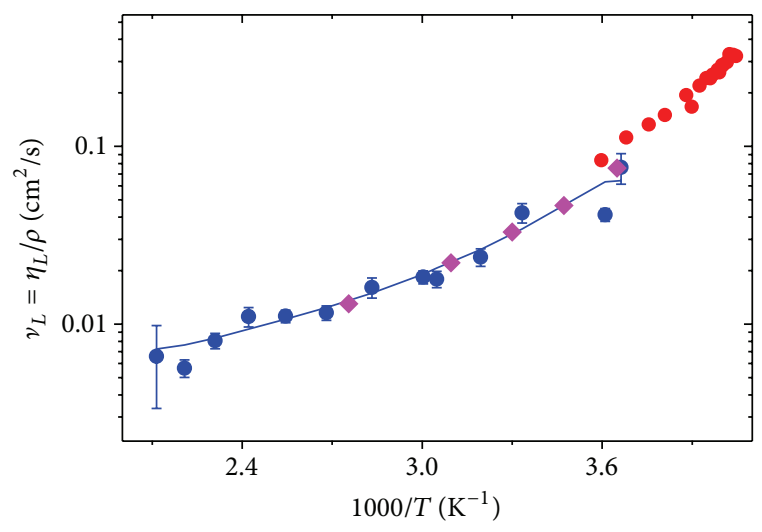

FIGURE 16: The Arrhenius plot of longitudinal viscosity of water as determined by (i) $Q=0$ extrapolation IXS results from [13] (blue dots); (ii) shear viscosity data multiplied by a factor 4 from [38] (blue line); (iii) BLS data in the supercooled region [7] (red circles); and (iv) US measurements [39] (purple diamonds).

Ts. A low value of $\eta_{\infty}$ seems also to be congruous with the unusually narrow width of water spectra suggested by both INS [25] and IXS $[9,12]$ measurements.

Most importantly, in nearly all measurements, $\eta_{\infty}$ was found to be much lower than the macroscopic shear viscosity $\eta_{s}$. This would imply that the relaxation observed in water involves not only the bulk, but also the shear component of viscosity and, likely, a large part of it. The same conclusion is also suggested by the observed parallelism in the $T$-dependencies of longitudinal and shear components of viscosity. An almost fully relaxing shear viscosity seems to be consistent with the peculiar ability of water to support shear propagation at moderate Qs. This topic will be discussed in further detail in a forthcoming publication.

\section{Toward Supercritical Conditions}

Among various results, [13] also demonstrated the gradual decrease of the $Q=0$ extrapolated relaxation strength $\left(\Delta^{2}=\Delta^{2}(Q=0)\right)$ upon approaching supercritical conditions, likely owing to the corresponding reduction in the number of HBs per molecule. This trend is shown in Figure 17 where IXS results in [13] are compared with US ones on aqueous solution of glycerol extrapolated in the limit of zero glycerol concentration [37]. In spite of the disparate frequencies covered by the two spectroscopic methods, an overall good agreement can be readily appreciated. Most importantly, one readily notices that $\Delta^{2}$ tend to vanish upon increasing $T$, even if error bars at high temperature become substantially larger. Although the critical temperature $T_{c}=647 \mathrm{~K}$ is not reached by plotted data, the systematic decrease of the amplitude $\Delta^{2}$ seems to suggest that any signature of the structural relaxation disappears upon approaching nearly critical or higher Ts.

This result was later confirmed by a more comprehensive IXS measurement [40], in which relaxation phenomena were comparatively investigated in few prototypical liquids having an increasing microscopic complexity: a monatomic 


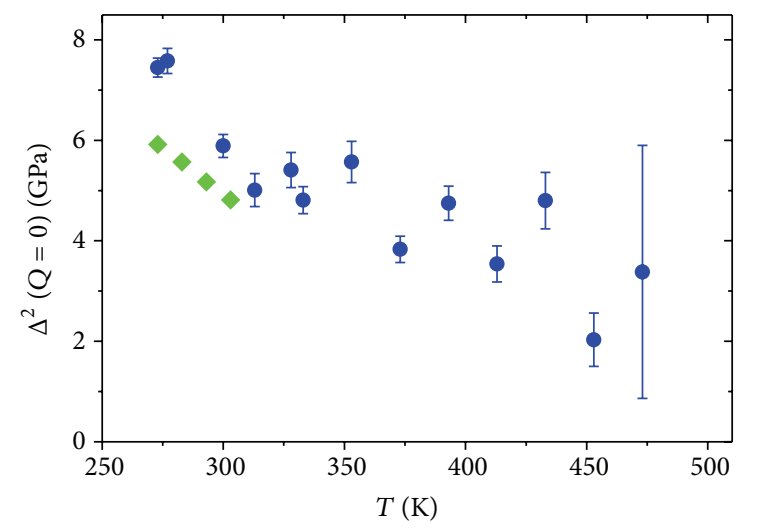

FIGURE 17: The temperature dependence of the $Q=0$ extrapolated relaxation strength derived from [13] (solid circles) is compared with the corresponding variable determined by US measurements [37].

fluid (neon), a diatomic fluid (nitrogen), an associated fluid (ammonia) and, finally, an associated fluid with clear thermodynamic anomalies, as liquid water.

In all cases the line-shape model was based on the memory function expressed by (10), in which, however, the parameters relevant to the thermal contribution were kept fixed to the values extracted from the NIST thermodynamic database see the NIST Chemistry WebBook: http://webbook.nist.gov/chemistry/form-ser.html.

Furthermore, in [40] an exponential $Q$ decay was used to extrapolate the macroscopic, $Q=0$, value of $\tau_{c}(Q)$. The extrapolated values, $\tau_{c}$, are plotted in Figure 18 as a function of the dimensionless variable $T_{c} / T$, with $T_{c}$ being the critical temperature.

When comparing the data obtained for non-associated (neon and nitrogen) and associated (water and ammonia) systems a striking difference readily emerges: while the former do not seem to depend on $T$ at all, the latter exhibits a sharp $T$-dependence at subcritical temperatures. At these low $T$ s the dominant relaxation processes acquire a clearly structural character as indicated by a sharp $T$-dependence expectedly parallel to the one of viscosity.

To perform a more meaningful comparison between the relaxation times of systems so diverse from each other, the authors considered a data normalization using a parameter taking into account the different thermodynamic conditions and molecular shapes of the samples. This was identified with the intermolecular collision period, $\tau_{\text {coll }}$, a parameter easily estimated by modeling the sample as a simple hard-sphere Boltzmann gas:

$$
\tau_{\text {coll }}=\frac{1}{\rho} \sqrt{\frac{1}{16 \pi d^{4} M k_{B} T}}
$$

where $d$ represents the molecular diameter.

In Figure 19 the normalized relaxation time $\tau / \tau_{\text {coll }}$ is reported as measured in [40] as a function of the dimensionless variable $T_{c} / T$. The first clear effect of the normalization is that around and above $T_{c}$ the absolute value of $\tau / \tau_{\text {coll }}$ becomes

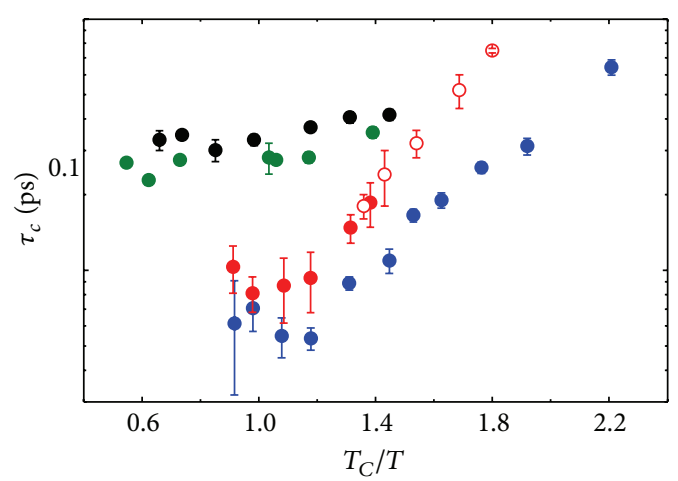

FIGURE 18: The $Q=0$ extrapolated relaxation timescale measured by IXS in [40] for several prototypical fluids as on neon (black dots), nitrogen (olive dots), ammonia (red dots), and water (blue dots). The relaxation times of ammonia extracted from the IXS work in [41] are also reported for comparison as open dots.

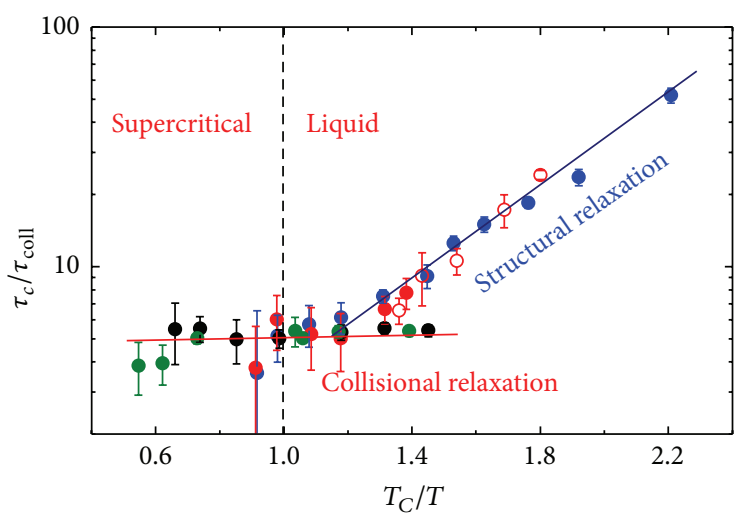

FIgURE 19: The same data as in Figure 18 are here rescaled for the corresponding collisional relaxation time (see (11)). The straight lines through the data serve as guide to eye. The vertical dashed line indicates the critical temperature.

essentially system-independent and well approximated by a horizontal line.

This implies that for temperature approaching $T_{c}$ or higher the relaxation time becomes proportional to the intercollision period, which indicates the essentially collisional nature of relaxation process at nearly critical or supercritical conditions.

Furthermore, Figure 19 shows that for subcritical Ts the dynamics of the two hydrogen bonded systems, ammonia and water, are dominated by a relaxation phenomenon, for which the sharply $T$-dependent timescale reveals a clearly structural origin.

Structural relaxations involve collective rearrangement of the local structure in response to an applied perturbation. They are dominating at subcritical $T$ s and in system having extended networks of intermolecular bonds. Furthermore, being these processes hampered by viscous resistance, their timescale exhibits in a first approximation the same dependence on thermodynamic conditions as the viscosity. 
Conversely, at supercritical conditions, relaxation processes essentially couple with intermolecular collisions, which become the dominating intermolecular interaction, since HBs decrease in both number and strength.

\section{Still Open Questions on the Dynamics of Supercooled Water}

In spite of the thorough investigations performed by both $\mathrm{X}$ ray and neutron probes, several questions are still open on the behavior of density fluctuations in deeply supercooled water. In fact, some thermodynamic properties related to density fluctuations, as for example, the isothermal compressibility and the thermal expansion coefficient, seemingly diverge upon approaching a temperature close to the homogeneous nucleation temperature $T_{h}$ [103] $\left(T_{h} \approx 228 \mathrm{~K}\right.$ at ambient pressure). The $P$-evolution of $T_{h}$ and the melting curve of $\mathrm{D}_{2} \mathrm{O}$ have been measured in [42] and the results are reported in the phase diagram of $\mathrm{D}_{2} \mathrm{O}$ in Figure 20.

Several models were developed in the attempt of explaining this anomalous behavior of density fluctuations.

Three alternative scenarios are currently invoked to account for this effect; they either assume the existence of a retracing spinodal [104], or that of a second critical point $[105,106]$, or, finally, propose a singularity-free explanation $[107,108]$. These scenarios should also provide a coherent explanation of analogous anomalies related to energy fluctuations, as those observed in constant volume or constant pressure specific heats [109]. However, a comprehensive theory providing a consistent account of all observed anomalies is lacking; furthermore, all experimental evidences attempting to validate any of these hypotheses have been thus far inconclusive [2].

Another open question related to supercooled water concerns the divergence, around the same temperature, of transport coefficients and, in particular, of shear and bulk viscosities [3, 39, 69]. However, it is still a matter of a controversy whether this divergence is critical-like or if it instead parallels a Vogel-Tammann-Fulcher law, as typical of glass-forming system.

As to be expected, the anomalous viscosity enhancement of supercooled water is reflected by a similar trend of the structural relaxation time. In Figure 21 the temperature dependence of the relaxation frequency $1 / \tau$ is reported as measured in [7] and [34] by BLS and BUVS, respectively, along with the straight line best fitting supercooled phase data. It clearly appears that $1 / \tau$ tends to vanish at a temperature somehow close to $228 \mathrm{~K}$ [7], which roughly corresponds the known value of $T_{h}$ as deduced, for example, from Figure 20.

A more recent result obtained using optical Kerr effect (OKE) methods [8] is also reported in the same plot for comparison. It clearly points to the presence of a structural arrest, that is, a diverging relaxation time, close to the hypothesized temperature of second critical point of water $(\approx 221 \mathrm{~K}[106])$.

According to [8], the temperature dependence of the relaxation time would follow a critical-like divergence of the form $\tau \propto\left(T-T_{c}\right)^{-\gamma}$ with $\gamma=2.2 \pm 0.3$ and $T_{c}=(221 \pm 5) \mathrm{K}$.

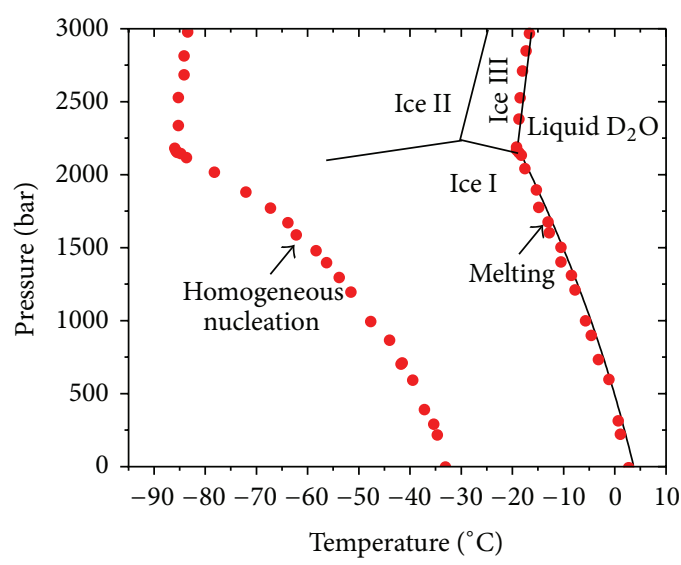

FIGURE 20: The phase diagram of heavy water is reported along with the melting and homogeneous nucleation curves measured in [42] (red dots)

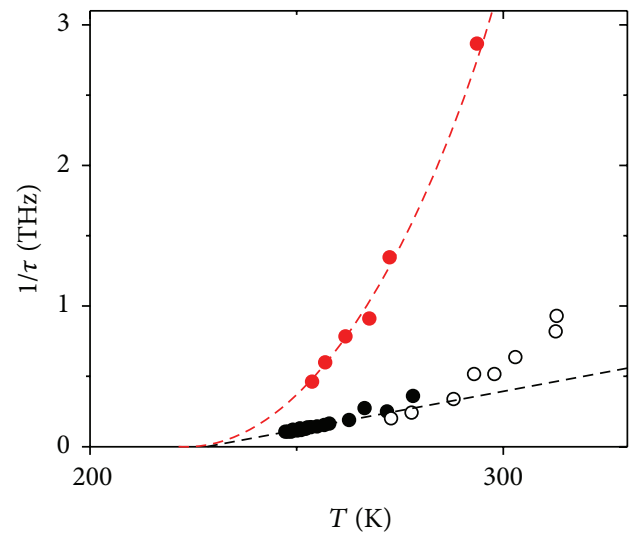

FIGURE 21: The relaxation frequency $1 / \tau$ of water measured in [7] (black dots) and [34] (open dots) are reported along with the linear best fit of supercooled phase data (black dashed line). Results obtained by OKE methods [8] are also reported for comparison (red dots) along with the critical law best fitting them (red dashed line).

In the foreseeable future, the use of $\mathrm{THz}$ spectroscopies such as IXS to study the dynamics of deeply supercooled water is deemed to shed a deeper insight into the signature of this divergence at mesoscopic scales, possibly clarifying its microscopic origin.

\section{The Contribution of Next Generation IXS Spectrometers}

The state-of-the-art IXS technique is held back from probing the dynamic response of deeply supercooled water by two major difficulties.

(1) The access to deep supercooling condition can be hardly achieved with large sized samples, owing to their likely nucleation (freezing). The use of water confined in nanoporous materials seems to be the most viable solution to this problem. However, IXS experiments on confined samples are in principle 
hampered by the large intensity scattering of sampleto-substrate interfaces. Owing to the broad and essentially Lorentzian wings of the energy resolution function, this scattering contribution overwhelms the low frequency inelastic signal of confined water, which makes its detection challenging.

(2) In the supercooled phase the dynamics of the sample becomes extremely slow and relaxation processes take place over timescale much longer than those in the window of sensitivity of current IXS instruments.

In order to effectively tackle these problems the development of next generation IXS instruments using a new monochromatization/energy analysis principle is required.

Most current IXS spectrometers use high-order Bragg reflections under extreme backscattering geometries, the energy analysis being often performed through scans of the temperature difference between the analyzer and monochromator crystals [110]. Unfortunately, the achievement of a meV resolution imposes demanding constraints on the temperature stability of active optical elements, since all temperature gradients must be $<10^{-4} \mathrm{~K}$. Furthermore, the state-of-the art IXS is hampered by the need of using high energy incident $\mathrm{X}$-ray beams, which penetrate more deeply into the crystals and are thus more severely affected by extinction losses.

In the foreseeable future, new concept optical schemes with the performance required to properly study the slow relaxations occurring in supercooled water can be developed. Few alternative schemes are currently considered for implementation and both exploit the dispersive nature of Bragg backreflection from asymmetrically cut crystals [111]: (1) an optical scheme consisting in an array of four Si crystals and customarily referred to as 4-bounce monochromator [112]; (2) an optical setup based on the simultaneous employment of a collimator (C), a dispersive (D), and a wavelength selector (W) crystal and referred to as CDW, or as CDDW if two dispersive crystals are used [113].

Both solutions were demonstrated to provide viable routes to the achievement of a sub-meV broad and extremely sharp resolution profiles. These optical schemes, based on multiple-bounce reflections from asymmetrically cut crystals, provide a wealth of advantages as compared to current backscattering IXS optics.

(1) They do not require the access to extremely high Bragg reflection orders, which would impose serious penalties to overall efficiency.

(2) The various bounces dramatically increase the spectral contrast, that is, the sharpness of the resolution function.

(3) These assemblies are ideally operated at moderate energies at which many current synchrotron undulators perform best.

(4) They offer a higher flexibility, including the opportunity of selecting nearly any energy and the capability of tailoring the resolution to any specific energy value.

The working principle of one of such schemes has been demonstrated in a recent work [114], which also shows, as an example, a sub-meV measurement of the IXS spectrum of glycerol. Most importantly, a novel beamline with a very narrow (sub-meV) and sharp (essentially Gaussian) resolution function [115] will be soon operative at the new synchrotron source NSLS II at Upton, NY.

\section{Concluding Remarks}

This paper reviews the advances in the knowledge of the high frequency viscoelastic behavior of water achieved over almost four decades of thorough spectroscopic investigations. The focus is on the collective dynamics of water in the socalled mesoscopic regime, corresponding to distances and timescales comparable with first neighbor separations and cage oscillation frequencies in liquid. A particular emphasis is on the contribution provided by inelastic X-ray scattering (IXS), a $\mathrm{THz}$ spectroscopic technique that, thanks to the virtual lack of kinematic limitation, has opened the access to previously unexplored dynamic regions.

Historically, the first and most controversial topic raised around the $\mathrm{THz}$ dynamic response of water focused on the presence of a "fast sound" mode in the spectrum of density fluctuations, namely, an acoustic mode propagating with a velocity similar to the speed of phonons in ice.

Not without some initial skepticism, the presence of such an excitation was ascribed to the onset of large viscoelastic effects induced by the interaction with a structural relaxation process. From the experimental side, this conclusion has been mostly reached thanks to the development of IXS spectrometers with energy resolution bandwidth of about $1 \mathrm{meV}$. In fact, this technique, owing to the virtual lack of kinematic limitations, has disclosed the access to dynamic region appropriate to study such relaxation phenomena.

In few years, rapid advances in X-ray sources and crystal optics have substantially improved the proficiency of IXS experiments in the line-shape acquisition and modeling. This has permitted us to unambiguously demonstrate that the relaxation active in water has a characteristic time, $\tau$, whose sharp temperature dependence has an Arrhenius form corresponding to an activation energy roughly matching the value hydrogen bonds ( $\mathrm{HB}$ ) energy of water. This pointed to a close link between the relaxation and the continuous breaking and forming of HBs.

The presence of this relaxation causes acoustic propagation in water to strongly depend on frequency and more specifically to depend on how the frequency compares with the relaxation timescales. Acoustic modes with frequencies much smaller than $1 / \tau$ propagate over a local structure which they essentially "perceive" as liquid-like, that is, rearranging much faster than the slow acoustic oscillations. In this viscous regime acoustic propagation is merely liquid-like; that is, it is characterized by a relatively low speed and large damping and density fluctuations do not couple with shear waves.

In the opposite, elastic regime modes with frequency much larger than $1 / \tau$ are generated by the probe and they "perceive" the propagating medium as frozen or solid-like. At these frequencies internal movements of the molecule are definitely too slow to efficiently couple with the rapidly oscillating acoustic wave, which therefore travels elastically, 
that is, with virtually no energy loss. In this elastic regime the response of the sample is essentially solid-like as typical of the glassy phase, that is characterized by a high sound velocity and a low sound damping, as well as by a sizable coupling between density fluctuations and transverse waves.

The scenario drastically changes when supercritical temperatures are reached. Here the active relaxation tends to lose its structural character, that is, its connection with $\mathrm{HB}$ network rearrangements. As a likely consequence of the reduction in the number of $\mathrm{HBs}$ per molecules, relaxation processes become increasingly coupled with intermolecular collisions, similar to what expected for a dense hard-sphere gas. Finally, in the metastable supercooled phase, both mesoscopic structure and dynamics of water bear evidence of an anomalous divergence around a temperature not far from the one hypothesized for a second critical point. At present, the investigation of this thermodynamic region is deemed to shed a deeper insight into still unknown and fascinating topics such as the effects of such a divergence at mesoscopic scales. However, the state-of-the-art $\mathrm{THz}$ spectroscopy is clearly held back from achieving this goal by inherent limitations in the instrumental resolution function. New advances in the field require the development of novel inelastic X-ray scattering spectrometers with narrower (sub-meV) energy bandwidth and sharper (nearly Gaussian) instrumental resolution function. A great hope is put on a new generation IXS spectrometer to be soon operative at the new synchrotron source NSLS II at Brookhaven National Laboratory. Its development is deemed to disclose new avenues on the study of relaxation phenomena in water and in more complex fluids.

\section{Conflict of Interests}

The author declares that there is no conflict of interests regarding the publication of this paper.

\section{Acknowledgments}

The whole IXS Team at Beamline ID16 is acknowledged for having performed all IXS measurements presented throughout the paper, for the modeling and interpretation of results and for the innumerable fruitful discussions. Also, the author feels deeply indebted to T. Rowell-Cunsolo for the critical revision of the paper.

\section{References}

[1] V. F. Petrenko and R. W. Whitworth, Physics of Ice, Oxford University Press, Oxford, UK, 1999.

[2] P. G. Debenedetti, Metastable Liquids: Concepts and Principles, Princeton University Press, Princeton, NJ, USA, 1996.

[3] K. E. Bett and J. B. Cappi, "Effect of pressure on the viscosity of water," Nature, vol. 207, no. 4997, pp. 620-621, 1965.

[4] F. X. Prielmeier, E. W. Lang, R. J. Speedy, and H.-D. Lüdemann, "Diffusion in supercooled water to $300 \mathrm{MPa}$," Physical Review Letters, vol. 59, no. 10, pp. 1128-1131, 1987.

[5] A. Cunsolo, A. Orecchini, C. Petrillo, and F. Sacchetti, "Quasielastic neutron scattering investigation of the pressure dependence of molecular motions in liquid water," Journal of Chemical Physics, vol. 124, no. 8, Article ID 084503, 2006.

[6] C. A. Angell, "Water II is a 'strong' liquid," The Journal of Physical Chemistry, vol. 97, no. 24, pp. 6339-6341, 1993.

[7] A. Cunsolo and M. Nardone, "Velocity dispersion and viscous relaxation in supercooled water," The Journal of Chemical Physics, vol. 105, no. 10, pp. 3911-3917, 1996.

[8] R. Torre, P. Bartolini, and R. Righini, "Structural relaxation in supercooled water by time-resolved spectroscopy," Nature, vol. 428, no. 6980, pp. 296-299, 2004.

[9] F. Sette, G. Ruocco, M. Krisch et al., "Collective dynamics in water by high energy resolution inelastic X-Ray scattering," Physical Review Letters, vol. 75, no. 5, pp. 850-853, 1995.

[10] S. C. Santucci, D. Fioretto, L. Comez, A. Gessini, and C. Masciovecchio, "Is there any fast sound in water?" Physical Review Letters, vol. 97, no. 22, Article ID 225701, 2006.

[11] G. Ruocco, F. Sette, U. Bergmann et al., "Equivalence of the sound velocity in water and ice at mesoscopic wavelengths," Nature, vol. 379, no. 6565, pp. 521-523, 1996.

[12] F. Sette, G. Ruocco, M. Krisch, C. Masciovecchio, R. Verbeni, and U. Bergmann, "Transition from normal to fast sound in liquid water," Physical Review Letters, vol. 77, no. 1, pp. 83-86, 1996.

[13] G. Monaco, A. Cunsolo, G. Ruocco, and F. Sette, "Viscoelastic behavior of water in the terahertz-frequency range: an inelastic X-ray scattering study," Physical Review E, vol. 60, no. 5, part A, pp. 5505-5521, 1999.

[14] A. Cunsolo, G. Ruocco, F. Sette et al., "Experimental determination of the structural relaxation in liquid water," Physical Review Letters, vol. 82, no. 4, pp. 775-778, 1999.

[15] A. Rahman and F. H. Stillinger, "Propagation of sound in water. A molecular-dynamics study," Physical Review A, vol. 10, no. 1, pp. 368-378, 1974.

[16] A. Rahman and F. H. Stillinger, "Molecular dynamics study of liquid water," The Journal of Chemical Physics, vol. 55, no. 7, pp. 3442-3449, 1971.

[17] D. Levesque, L. Verlet, and J. Kürkijarvi, "Computer "experiments" on classical fluids. IV. Transport properties and timecorrelation functions of the Lennard-Jones liquid near its triple point," Physical Review A, vol. 7, no. 5, pp. 1690-1700, 1973.

[18] I. M. de Schepper, J. C. van Rijs, A. A. van Well, P. Verkerk, L. A. de Graaf, and C. Bruin, "Microscopic sound waves in dense Lennard-Jones fluids," Physical Review A, vol. 29, no. 3, pp. 1602-1605, 1984.

[19] C. Bruin, J. C. van Rijs, L. A. de Graaf, and I. M. de Schepper, "Density dependence of sound dispersion in repulsive LennardJones fluids," Physical Review A, vol. 34, no. 4, pp. 3196-3202, 1986.

[20] W. E. Alley, B. J. Alder, and S. Yip, "The neutron scattering function for hard spheres," Physical Review A, vol. 27, no. 6, pp. 3174-3186, 1983.

[21] R. W. Impey, P. A. Madden, and I. R. McDonald, "Spectroscopic and transport properties of water: model calculations and the interpretation of experimental results," Molecular Physics, vol. 46, no. 3, pp. 513-539, 1982.

[22] O. Matsuoka, E. Clementi, and M. Yoshimine, "Cl study of the water dimer potential surface," The Journal of Chemical Physics, vol. 64, no. 4, pp. 1351-1361, 1976.

[23] G. E. Walrafen, "Raman and infrared spectral investigations of water structure," in Water: A Comprehensive Treatise, vol. 1, pp. 151-214, Plenum, New York, NY, USA, 1972. 
[24] P. Bosi, F. Dupré, F. Menzingee, F. Sacchetti, and M. C. Spinelli, "Observation of collective excitations in heavy water in the 108 $\mathrm{cm}^{-1}$ momentum range," Lettere al Nuovo Cimento, vol. 21, no. 12, pp. 436-440, 1978.

[25] J. Teixeira, M. C. Bellissent-Funel, S. H. Chen, and B. Dorner, "Observation of new short-wavelength collective excitations in heavy water by coherent inelastic neutron scattering," Physical Review Letters, vol. 54, no. 25, pp. 2681-2683, 1985.

[26] F. Demichelis, A. Gordana, and A. Tartaglia, "Molecular dynamics in $\mathrm{H}_{2} \mathrm{O}$ and $\mathrm{D}_{2} \mathrm{O}$ analysis by inelastic-scattering of 10 Å neutrons," Nuovo Cimento B, vol. 37, no. 2, pp. 185-197, 1977.

[27] U. Balucani and M. Zoppi, Dynamics of the Liquid State, Clarendon Press, Oxford, UK, 1994.

[28] H. Bell, H. Moeller-Wenghoffer, A. Kollmar, R. Stockmeyer, T. Springer, and H. Stiller, "Neutron Brillouin scattering in fluid neon," Physical Review A, vol. 11, no. 1, pp. 316-327, 1975.

[29] J. R. D. Copley and J. M. Rowe, "Short-wavelength collective excitations in liquid rubidium observed by coherent neutron scattering," Physical Review Letters, vol. 32, no. 2, pp. 49-52, 1974.

[30] O. Soderstrom, J. R. D. Copley, J.-B. Suck, and B. Dorner, "Collective excitations in liquid lead," Journal of Physics F, vol. 10, no. 6, pp. L151-L158, 1980.

[31] F. J. Bermejo, M. Alvarez, S. M. Bennington, and R. Vallauri, "Absence of anomalous dispersion features in the inelastic neutron scattering spectra of water at both sides of the melting transition," Physical Review E, vol. 51, no. 3, pp. 2250-2262, 1995.

[32] A. Giugni and A. Cunsolo, "Structural relaxation in the dynamics of glycerol: a joint visible, UV and x-ray inelastic scattering study," Journal of Physics Condensed Matter, vol. 18, no. 3, pp. 889-902, 2006.

[33] J.-C. Bacri and R. Rajaonarison, "Etude ultrasonore de l'eau en surfusion jusqu'à $-26^{\circ}$ C, J Journal de Physique Lettres, vol. 40, no. 16, pp. 403-405, 1979.

[34] F. Bencivenga, A. Cimatoribus, A. Gessini, M. G. Izzo, and C. Masciovecchio, "Temperature and density dependence of the structural relaxation time in water by inelastic ultraviolet scattering," The Journal of Chemical Physics, vol. 131, no. 14, Article ID 144502, 2009.

[35] V. Mazzacurati, A. Nucara, M. A. Ricci, G. Ruocco, and G. Signorelli, "High-resolution low-frequency Raman spectra of liquid $\mathrm{H}_{2} \mathrm{O}$ and $\mathrm{D}_{2} \mathrm{O}$," The Journal of Chemical Physics, vol. 93, no. 11, pp. 7767-7773, 1990.

[36] A. Fontana, M. Nardone, and M. A. Ricci, "Depolarized Rayleigh scattering in water up to supercritical conditions," The Journal of Chemical Physics, vol. 102, no. 18, pp. 6975-6981, 1995.

[37] W. M. Sli, A. R. Donfok Jr., and T. A. Litovitz, "Ultrasonic shear and longitudinal measurements in aqueous glycerol," The Journal of Chemical Physics, vol. 44, no. 10, pp. 3712-3718, 1965.

[38] J. Kestin, J. V. Sengers, B. Kamgar-Parsi, and J. M. H. Levelt Sengers, "Thermophysical properties of fluid $\mathrm{H}_{2} \mathrm{O}$," Journal of Physical and Chemical Reference Data, vol. 13, no. 1, article 175, 1984.

[39] C. M. Davis and J. Jarzynsky, "Liquid water-acoustic properties: absorption and relaxation," in Water: A Comprehensive Treatise, vol. 1, pp. 443-461, Plenum, New York, NY, USA, 1972.

[40] F. Bencivenga, A. Cunsolo, M. Krisch, G. Monaco, G. Ruocco, and F. Sette, "High frequency dynamics in liquids and supercritical fluids: a comparative inelastic X-Ray Scattering Study," Journal of Chemical Physics, vol. 130, no. 6, Article ID 064501, 2009.
[41] P. Giura, R. Angelini, F. Datchi, G. Ruocco, and F. Sette, "High frequency dynamics and structural relaxation process in liquid ammonia," Journal of Chemical Physics, vol. 127, no. 8, Article ID 084508, 2007.

[42] C. A. Angell and J. C. Tucker, "Anomalous heat capacities of supercooled water and heavy water," Science, vol. 181, no. 4097, pp. 342-344, 1973.

[43] Y. H. Jeong, S. R. Nagel, and S. Bhattacharya, "Ultrasonic investigation of the glass transition in glycerol," Physical Review A, vol. 34, no. 1, pp. 602-608, 1986.

[44] M. Wojcik and E. Clementi, "Collective dynamics in three body water and sound dispersion," The Journal of Chemical Physics, vol. 85, no. 10, pp. 6085-6092, 1986.

[45] M. A. Ricci, D. Rocca, G. Ruocco, and R. Vallauri, "Collective dynamical properties of liquid water," Physical Review Letters, vol. 61, no. 17, pp. 1958-1961, 1988.

[46] J. Bosse, G. Jacucci, M. Ronchetti, and W. Schirmacher, "Fast sound in two-component liquids," Physical Review Letters, vol. 57, no. 26, pp. 3277-3279, 1986.

[47] A. Campa and E. G. D. Cohen, "Observable fast kinetic eigenmode in binary noble-gas mixtures?" Physical Review Letters, vol. 61, no. 7, pp. 853-856, 1988.

[48] A. Campa and E. G. D. Cohen, "Kinetic-sound propagation in dilute gas mixtures," Physical Review A, vol. 39, no. 9, pp. 49094911, 1989.

[49] W. L. Jorgensen, J. Chandrasekhar, J. D. Madura, R. W. Impey, and M. L. Klein, "Comparison of simple potential functions for simulating liquid water," The Journal of Chemical Physics, vol. 79, no. 2, pp. 926-935, 1983.

[50] M. A. Ricci, D. Rocca, G. Ruocco, and R. Vallauri, “Theoretical and computer-simulation study of the density fluctuations in liquid water," Physical Review A, vol. 40, no. 12, pp. 7226-7238, 1989.

[51] E. Trinh and R. E. Apfel, "Sound velocity of supercooled water down to $-33 \circ \mathrm{C}$ using acoustic levitation," The Journal of Chemical Physics, vol. 72, no. 12, pp. 6731-6735, 1980.

[52] J. Rouch, C. C. Lai, and S. H. Chen, "Brillouin scattering studies of normal and supercooled water," The Journal of Chemical Physics, vol. 65, no. 10, pp. 4016-4021, 1976.

[53] J. Rouch, C. C. Lai, and S.-H. Chen, "High frequency sound velocity and sound absorption in supercooled water and the thermodynamic singularity at $228 \circ \mathrm{K}$," The Journal of Chemical Physics, vol. 66, no. 11, pp. 5031-5034, 1977.

[54] L. Bosio, J. Teixeira, and H. E. Stanley, "Enhanced density fluctuations in supercooled $\mathrm{H}_{2} \mathrm{O}, \mathrm{D}_{2} \mathrm{O}$, and ethanol-water solutions: evidence from small-angle X-ray scattering," Physical Review Letters, vol. 46, no. 9, pp. 597-600, 1981.

[55] Y. Xie, K. F. Ludwig Jr., G. Morales, D. E. Hare, and C. M. Sorensen, "Noncritical behavior of density fluctuations in supercooled water," Physical Review Letters, vol. 71, no. 13, pp. 2050-2053, 1993.

[56] G. N. I. Clark, G. L. Hura, J. Teixeira, A. K. Soper, and T. HeadGordon, "Small-angle scattering and the structure of ambient liquid water," Proceedings of the National Academy of Sciences of the United States of America, vol. 107, no. 32, pp. 14003-14007, 2010.

[57] A. Cunsolo, F. Formisano, C. Ferrero, F. Bencivenga, and S. Finet, "Pressure dependence of the large-scale structure of water," The Journal of Chemical Physics, vol. 131, no. 19, Article ID 194502, 2009. 
[58] U. Balucani, G. Ruocco, A. Torcini, and R. Vallauri, "Fast sound in liquid water," Physical Review E, vol. 47, no. 3, pp. 1677-1684, 1993.

[59] F. Sciortino and S. Sastry, "Sound propagation in liquid water: the puzzle continues," The Journal of Chemical Physics, vol. 100, no. 5, pp. 3881-3893, 1994.

[60] S. Magazú, G. Maisano, D. Majolino et al., "Relaxation process in deeply supercooled water by Mandelstam-Brillouin scattering," The Journal of Physical Chemistry, vol. 93, no. 2, pp. 942947, 1989.

[61] G. Maisano, P. Migliardo, F. Aliotta, C. Vasi, F. Wanderlingh, and G. D'Arrigo, "Evidence of anomalous acoustic behavior from Brillouin scattering in supercooled water," Physical Review Letters, vol. 52, no. 12, pp. 1025-1028, 1984.

[62] O. Conde, J. Leblond, and J. Teixeira, "Analysis of the dispersion of the sound velocity in supercooled water," Journal de Physique Paris, vol. 41, no. 9, pp. 997-1000, 1980.

[63] P. A. Fleury and J. P. Boon, "Brillouin scattering in simple liquids: argon and neon," Physical Review, vol. 186, no. 1, pp. 244-254, 1969.

[64] J. P. Boon and S. Yip, Molecular Hydrodynamics, Courier Dover Publications, 1980.

[65] J. A. Saxton, "Dielectric dispersion in pure polar liquids at very high radio-frequencies. II. Relation of experimental results to theory," Proceedings of the Royal Society A, vol. 213, no. 1115, pp. 473-492, 1952.

[66] J. B. Hasted, "Liquid water: dielectric properties," in Water: A Comprehensive Treatise, F. Franks, Ed., vol. 1, pp. 255-309, Plenum, New York, NY, USA, 1972.

[67] T. A. Litovitz and G. E. McDuffie Jr., "Comparison of dielectric and mechanical relaxation in associated liquids," The Journal of Chemical Physics, vol. 39, no. 3, pp. 729-734, 1963.

[68] K. J. Laidler, Chemical Kinetics, Harper \& Row, New York, NY, USA, 1987.

[69] J. Hallett, "The temperature dependence of the viscosity of supercooled water," Proceedings of the Physical Society, vol. 82, no. 6, pp. 1046-1050, 1963.

[70] M. Krisch and F. Sette, Neutron and X Ray Spectroscopy, Springer, Berlin, Germany, 2007.

[71] G. Ruocco and F. Sette, "The history of the 'fast sound' in liquid water," Condensed Matter Physics, vol. 11, no. 1, pp. 29-46, 2008.

[72] B. Renker, "Phonon dispersion in $\mathrm{D}_{2} \mathrm{O}$-ice," Physics Letters A, vol. 30, no. 9, pp. 493-494, 1969.

[73] E. Pontecorvo, M. Krisch, A. Cunsolo et al., "High-frequency longitudinal and transverse dynamics in water," Physical Review E-Statistical, Nonlinear, and Soft Matter Physics, vol. 71, no. 1, Article ID 011501, 2005.

[74] A. Cimatoribus, S. Saccani, F. Bencivenga, A. Gessini, M. G. Izzo, and C. Masciovecchio, "The mixed longitudinaltransverse nature of collective modes in water," New Journal of Physics, vol. 12, Article ID 053008, 2010.

[75] A. Cunsolo, C. N. Kodituwakku, F. Bencivenga, M. Frontzek, B. M. Leu, and A. H. Said, "Transverse dynamics of water across the melting point: a parallel neutron and $\mathrm{x}$-ray inelastic scattering study," Physical Review B: Condensed Matter and Materials Physics, vol. 85, no. 17, Article ID 174305, 2012.

[76] A. Cunsolo, "Onset of a transverse dynamics in the $\mathrm{THz}$ spectrum of water," Molecular Physics, vol. 111, no. 3, pp. 455463, 2013

[77] M. Sampoli, G. Ruocco, and F. Sette, "Mixing of longitudinal and transverse dynamics in liquid water," Physical Review Letters, vol. 79, no. 9, pp. 1678-1681, 1997.
[78] R. D. Mountain, "Spectral distribution of scattered light in a simple fluid," Reviews of Modern Physics, vol. 38, no. 1, pp. 205214, 1966.

[79] K. F. Herzfeld and T. A. Litovitz, Absorption and Dispersion of Ultrasonic Waves, Pure and Applied Physics, Vol. 7, Academic Press, London, UK, 1965.

[80] U. Bafile, E. Guarini, and F. Barocchi, "Collective acoustic modes as renormalized damped oscillators: unified description of neutron and x-ray scattering data from classical fluids," Physical Review E-Statistical, Nonlinear, and Soft Matter Physics, vol. 73, no. 6, Article ID 061203, 2006.

[81] F. Bencivenga, A. Cunsolo, M. Krisch et al., "Structural and collisional relaxations in liquids and supercritical fluids," Physical Review Letters, vol. 98, no. 8, Article ID 085501, 2007.

[82] R. Zwanzig, Statistical Mechanics of Irreversibility, vol. 3 of Lectures in Theoretical Physiscs, edited by W. Brittin, WileyInterscience, New York, NY, USA, 1961.

[83] S. W. Lovesey, "Density fluctuations in classical monatomic liquids," Journal of Physics C: Solid State Physics, vol. 4, no. 18, pp. 3057-3064, 1971.

[84] P. Debye, “Zur Theorie der spezifischen Wärmen," Annalen der Physik, vol. 344, no. 14, pp. 789-839, 1912.

[85] D. Bertolini and A. Tani, "Stress tensor and viscosity of water: molecular dynamics and generalized hydrodynamics results," Physical Review E, vol. 52, no. 2, pp. 1699-1710, 1995.

[86] W. Gotze, Liquids, Freezing and the Glass Transition, edited by J. P. Hansen, D. Levesque, J. Zinn-Justin, North-Holland, Amsterdam, The Netherlands, 1991.

[87] D. Bertolini and A. Tani, "Thermal conductivity of water: Molecular dynamics and generalized hydrodynamics results," Physical Review E: Statistical Physics, Plasmas, Fluids, and Related Interdisciplinary Topics, vol. 56, no. 4, pp. 4135-4151, 1997.

[88] R. Angelini, P. Giura, G. Monaco, G. Ruocco, F. Sette, and R. Verbeni, "Structural and microscopic relaxation processes in liquid hydrogen fluoride," Physical Review Letters, vol. 88, no. 25, part 1, 4 pages, 2002.

[89] A. Cunsolo, G. Pratesi, R. Verbeni et al., "Microscopic relaxation in supercritical and liquid neon," Journal of Chemical Physics, vol. 114, no. 5, pp. 2259-2267, 2001.

[90] T. Scopigno, E. Pontecorvo, R. di Leonardo et al., "Highfrequency transverse dynamics in glasses," Journal of Physics Condensed Matter, vol. 15, no. 11, pp. S1269-S1278, 2003.

[91] F. Bencivenga, A. Cunsolo, M. Krisch, G. Monaco, G. Ruocco, and F. Sette, "Adiabatic and isothermal sound waves: the case of supercritical nitrogen," Europhysics Letters, vol. 75, no. 1, pp. 70-76, 2006.

[92] L. Comez, D. Fioretto, G. Monaco, and G. Ruocco, "Brillouin scattering investigations of fast dynamics in glass forming systems," Journal of Non-Crystalline Solids, vol. 307-310, pp. 148153, 2002.

[93] G. Ruocco, F. Sette, R. di Leonardo et al., "Relaxation processes in harmonic glasses?” Physical Review Letters, vol. 84, no. 25, pp. 5788-5791, 2000.

[94] R. Verbeni, A. Cunsolo, G. Pratesi et al., "Quantum effects in the dynamics of He probed by inelastic X-ray scattering," Physical Review E: Statistical, Nonlinear, and Soft Matter Physics, vol. 64, no. 2, part 1, 6 pages, 2001.

[95] A. Cunsolo, G. Monaco, M. Nardone, G. Pratesi, and R. Verbeni, "Transition from the collective to the single-particle regimes in a quantum fluid," Physical Review B: Condensed Matter and Materials Physics, vol. 67, no. 2, Article ID 024507, 8 pages, 2003. 
[96] A. Z. Akcasu and E. Daniels, "Fluctuation analysis in simple fluids," Physical Review A, vol. 2, no. 3, pp. 962-975, 1970.

[97] G. E. Walrafen, "Raman spectral studies of the effects of temperature on water and electrolyte solutions," The Journal of Chemical Physics, vol. 44, no. 4, pp. 1546-1558, 1966.

[98] G. E. Walrafen, "Raman spectral studies of water structure," The Journal of Chemical Physics, vol. 40, no. 11, pp. 3249-3256, 1964.

[99] G. S. Fulcher, "Analysis of recent measurements of the viscosity of glasses," Journal of the American Ceramic Society, vol. 8, no. 6, pp. 339-355, 1925.

[100] C. A. Angell, "Strong and fragile liquids," in Relaxations in Complex Systems, K. L. Ngai and G. B. Wright, Eds., pp. 3-11, NRL, Washington, DC, USA, 1985.

[101] P. Taborek, R. N. Kleiman, and D. J. Bishop, "Power-law behavior in the viscosity of supercooled liquids," Physical Review B, vol. 34, no. 3, pp. 1835-1840, 1986.

[102] S. Matsuoka, G. H. Fredrickson, and G. E. Johnson, "Application of Adam-Gibbs' theory to thermodynamic recovery and structural relaxation," in Molecular Dynamics and Relaxation Phenomena in Glasses, T. Dorfmüller and W. Williams, Eds., vol. 277 of Lecture Notes in Physics, pp. 188-202, Springer, Berlin, Germany, 1987.

[103] R. J. Speedy and C. A. Angell, "Isothermal compressibility of supercooled water and evidence for a thermodynamic singularity at $-45^{\circ} \mathrm{C}$," The Journal of Chemical Physics, vol. 65, no. 3, pp. 851-858, 1976.

[104] M. C. D’Antonio and P. G. Debenedetti, "Loss of tensile strength in liquids without property discontinuities: a thermodynamic analysis," The Journal of Chemical Physics, vol. 86, no. 4, pp. 2229-2235, 1987.

[105] P. H. Poole, F. Sciortino, U. Essmann, and H. E. Stanley, "Phase behaviour of metastable water," Nature, vol. 360, no. 6402, pp. 324-328, 1992.

[106] O. Mishima and H. E. Stanley, "The relationship between liquid, supercooled and glassy water," Nature, vol. 396, no. 6709, pp. 329-335, 1998.

[107] P. H. Poole, F. Sciortino, T. Grande, H. E. Stanley, and C. A. Angell, "Effect of hydrogen bonds on the thermodynamic behavior of liquid water," Physical Review Letters, vol. 73, no. 12, pp. 1632-1635, 1994.

[108] S. Sastry, P. G. Debenedetti, F. Sciortino, and H. E. Stanley, "Singularity-free interpretation of the thermodynamics of supercooled water," Physical Review E: Statistical Physics, Plasmas, Fluids, and Related Interdisciplinary Topics, vol. 53, no. 6, pp. 6144-6154, 1996.

[109] C. A. Angell, J. Shuppert, and J. C. Tucker, "Anomalous properties of supercooled water. Heat capacity, expansivity, and proton magnetic resonance chemical shift from 0 to $-38^{\circ}$," Journal of Physical Chemistry, vol. 77, no. 26, pp. 3092-3099, 1973.

[110] C. Masciovecchio, U. Bergmann, M. Krisch, G. Ruocco, F. Sette, and R. Verbeni, "A perfect crystal X-ray analyser with $1.5 \mathrm{meV}$ energy resolution," Nuclear Instruments and Methods in Physics Research, Section B: Beam Interactions with Materials and Atoms, vol. 117, no. 3, pp. 339-340, 1996.

[111] Y. Shvyd'ko, X-Ray Optics-High-Energy-Resolution Applications, Springer Series in Optical Sciences, Springer, Berlin, Germany, 2004.

[112] M. Yabashi, K. Tamasaku, S. Kikuta, and T. Ishikawa, "Xray monochromator with an energy resolution of $8 \times 10^{-9}$ at $14.41 \mathrm{keV}$,' Review of Scientific Instruments, vol. 72, no. 11, p. 4080, 2001.
[113] Y. V. Shvyda'ko, M. Lerche, U. Kuetgens, H. D. Rüter, A. Alatas, and J. Zhao, "X-ray Bragg diffraction in asymmetric backscattering geometry," Physical Review Letters, vol. 97, no. 23, Article ID 235502, 2006.

[114] Y. Shvyd'ko, S. Stoupin, D. Shu et al., "High-contrast submillivolt inelastic X-ray scattering for nano- and mesoscale science," Nature Communications, vol. 5, article 4219, 2014.

[115] Y. Q. Cai, D. S. Coburn, A. Cunsolo et al., "The ultrahigh resolution IXS beamline of NSLS-II: recent advances and scientific opportunities," Journal of Physics: Conference Series, vol. 425, no. 20, Article ID 202001, 2013. 

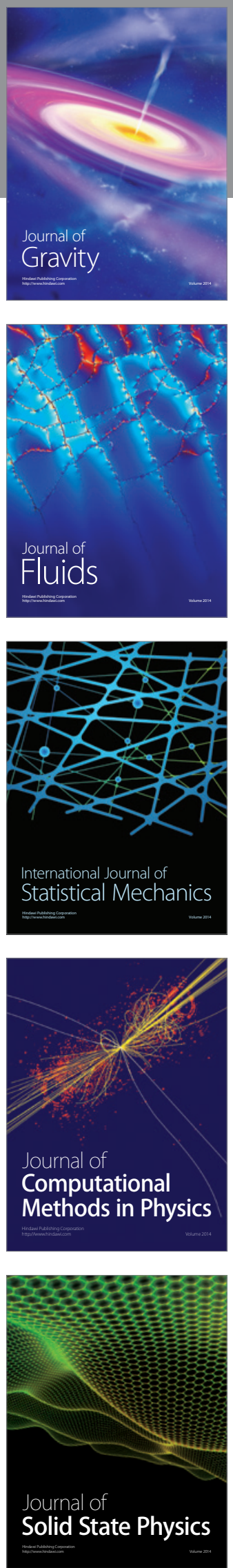

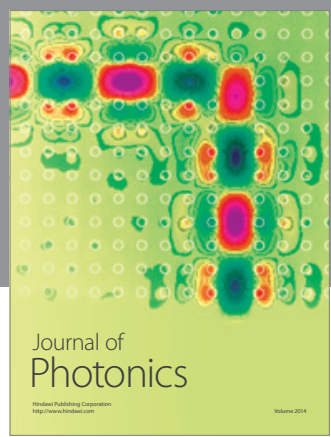

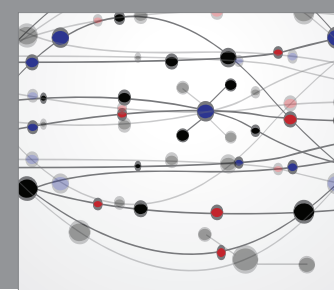

The Scientific World Journal

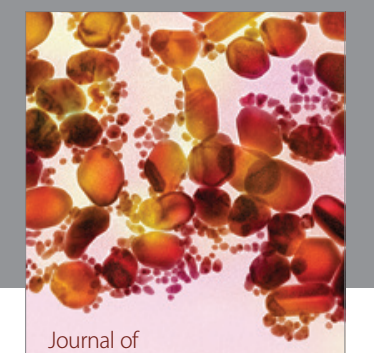

Soft Matter
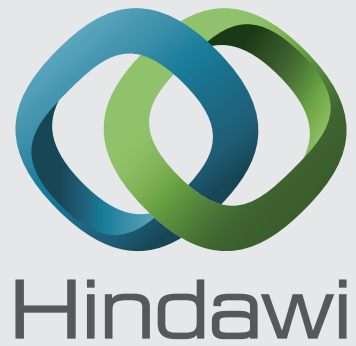

Submit your manuscripts at

http://www.hindawi.com
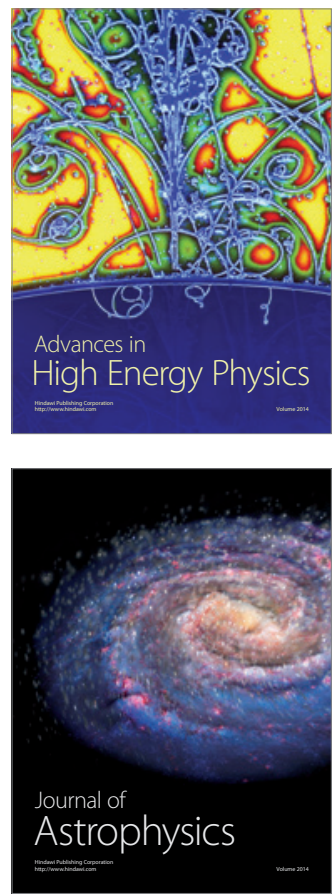
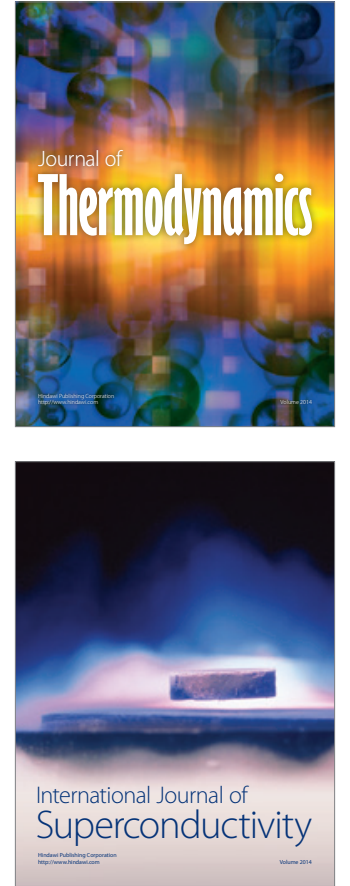
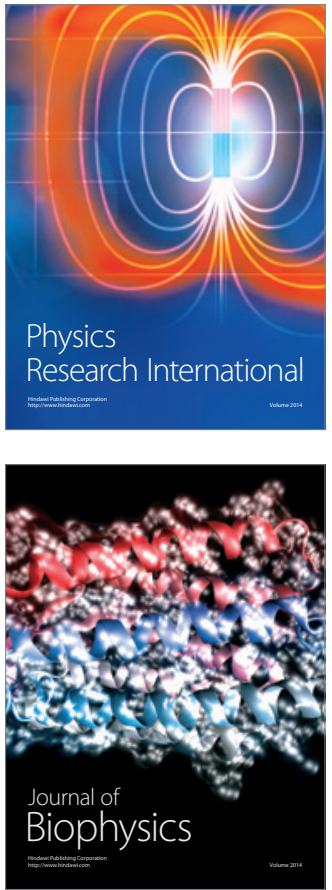
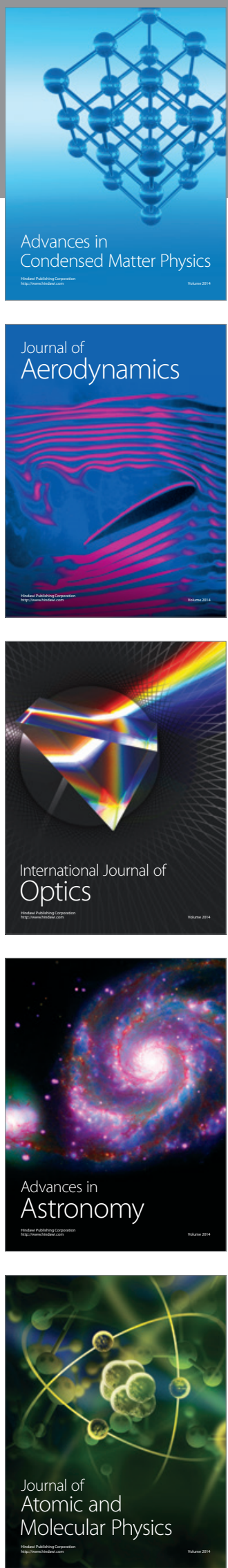